\title{
Multi-Colour Braid-Monoid Algebras
}

\author{
Uwe Grimm and Paul A. Pearce \\ Department of Mathematics, University of Melbourne, \\ Parkville, Victoria 3052, Australia
}

March 1993

\begin{abstract}
We define multi-colour generalizations of braid-monoid algebras and present explicit matrix representations which are related to two-dimensional exactly solvable lattice models of statistical mechanics. In particular, we show that the two-colour braid-monoid algebra describes the Yang-Baxter algebra of the critical dilute A-D-E models which were recently introduced by Warnaar, Nienhuis, and Seaton as well as by Roche. These and other solvable models related to dense and dilute loop models are discussed in detail and it is shown that the solvability is a direct consequence of the algebraic structure. It is conjectured that the Yang-Baxterization of general multi-colour braid-monoid algebras will lead to the construction of further solvable lattice models.
\end{abstract}

\section{Introduction}

The study of Yang-Baxter equations [1] has revealed a rich underlying algebraic structure in integrable systems with applications ranging from statistical mechanics to knot theory. Most well known among these algebraic structures is the quantum group. However, Yang-Baxter algebras are intimately connected to a number of other algebraic structures, most notably, the braid group [2] and the Temperley-Lieb [3] and Hecke algebras [4]. The braid and Temperley-Lieb or monoid [5] operators were first combined into a single algebra in 1987 by Birman and Wenzl [6] and independently by Murakami [7]. Subsequently, generalized braidmonoid algebras were introduced by Wadati, Deguchi and Akutsu [8]. On the one hand, these algebras are related to certain two-dimensional exactly solvable lattice models. On the other hand, the braid-monoid algebras admit a simple diagrammatic interpretation which points to the connections with the isotopy of knots and links. In this paper, we consider a generalization of the braid-monoid algebras where each strand or string of a link is assigned a colour. Some representations of these extended algebras correspond to the Yang-Baxter algebras of new critical solvable lattice models recently obtained by the Amsterdam group [9]. In particular, the two colour algebra is related to the dilute A-D-E models [10, [1].

The paper is organized as follows. We begin by defining the multi-colour braid-monoid algebra in sec. 2. A graphical interpretation is presented and a generalized notion of crossing symmetry is introduced. In sec. 3, we consider matrix representations of the multi-colour algebra and give explicit expressions for different types of representations. Sec. 4 concentrates on the two-colour case and the relation to exactly solvable models. The Yang-Baxter algebra of several RSOS models, vertex models, and mixed vertex-RSOS models is shown to be 
described by the two-colour algebra. Our main point, however, is that the algebraic structure is actually sufficient to guarantee that the Yang-Baxter equations are satisfied and hence yields the solvability of the models. We conclude by summarizing our results and give an outlook on possible consequences and further investigations. In particular, we conjecture that the general multi-colour braid-monoid can be Yang-Baxterized [12] to obtain new exactly solvable lattice models. Before proceeding, we point out that coloured braids and links have been considered previously by Akutsu, Deguchi and Wadati [13, 14, 15, 16, 17]. However, the developments of these papers are unrelated to the present paper.

\section{Definition of the Multi-Colour Algebra}

The $(n+1)$-string $m$-colour braid-monoid algebra is the associative algebra generated by the unit element $I$ (i.e., $I x=x I=x$ for all elements $x$ of the algebra), central elements $\sqrt{Q^{(\alpha)}}$ and $\omega^{(\alpha)}(1 \leq \alpha \leq m)$ together with three sets of generators

- $m(n+1)$ "projectors" $P_{j}^{(\alpha)}(1 \leq j \leq n+1,1 \leq \alpha \leq m)$

- $m^{2} n$ "coloured Temperley-Lieb operators" $e_{j}^{(\alpha, \beta)}$

- $m^{2} n$ "coloured braids" $b^{+}{ }_{j}^{(\alpha, \beta)}=b_{j}^{(\alpha, \beta)}$ and "coloured inverse braids" $b_{j}^{-(\alpha, \beta)}$

which fulfill the following list of relations

(i) projector relations:

$$
\begin{aligned}
P_{j}^{(\beta)} P_{j}^{(\alpha)} & =\delta_{\alpha, \beta} P_{j}^{(\alpha)} \\
P_{k}^{(\beta)} P_{j}^{(\alpha)} & =P_{j}^{(\alpha)} P_{k}^{(\beta)} \quad \text { for } j \neq k \\
\sum_{\alpha=1}^{m} P_{j}^{(\alpha)} & =I
\end{aligned}
$$

(ii) braid relations:

$$
\begin{aligned}
b_{j}^{-(\beta, \alpha)} b_{j}^{(\alpha, \beta)} & =b_{j}^{(\beta, \alpha)} b_{j}^{-(\alpha, \beta)}=P_{j}^{(\alpha)} P_{j+1}^{(\beta)} \\
b_{k}^{(\gamma, \delta)} b_{j}^{(\alpha, \beta)} & =b_{j}^{(\alpha, \beta)} b_{k}^{(\gamma, \delta)} \text { for }|j-k|>1 \\
b_{j+1}^{(\gamma, \alpha)} b_{j}^{(\gamma, \beta)} b_{j+1}^{(\alpha, \beta)} & =b_{j}^{(\alpha, \beta)} b_{j+1}^{(\gamma, \beta)} b_{j}^{(\gamma, \alpha)}
\end{aligned}
$$

(iii) Temperley-Lieb (monoid) relations:

$$
\begin{aligned}
e_{j}^{(\beta, \gamma)} e_{j}^{(\alpha, \beta)} & =\sqrt{Q^{(\beta)}} e_{j}^{(\alpha, \gamma)} \\
e_{k}^{(\gamma, \delta)} e_{j}^{(\alpha, \beta)} & =e_{j}^{(\alpha, \beta)} e_{k}^{(\gamma, \delta)} \quad \text { for }|j-k|>1 \\
e_{j}^{(\beta, \gamma)} e_{j \pm 1}^{(\beta, \beta)} e_{j}^{(\alpha, \beta)} & =e_{j}^{(\alpha, \gamma)} P_{j+(1 \pm 3) / 2}^{(\beta)}
\end{aligned}
$$


(iv) "twist" relations:

$$
\begin{aligned}
e_{k}^{(\gamma, \delta)} b_{j}^{(\alpha, \beta)} & =b_{j}^{(\alpha, \beta)} e_{k}^{(\gamma, \delta)} \quad \text { for }|j-k|>1 \\
b_{j}^{(\beta, \beta)} e_{j}^{(\alpha, \beta)} & =\omega^{(\beta)} e_{j}^{(\alpha, \beta)} \\
e_{j}^{(\alpha, \beta)} b_{j}^{(\alpha, \alpha)} & =\omega^{(\alpha)} e_{j}^{(\alpha, \beta)}
\end{aligned}
$$

(v) braid-monoid relations:

$$
\begin{aligned}
b_{j+1}^{(\gamma, \beta)} b_{j}^{(\gamma, \beta)} e_{j+1}^{(\alpha, \beta)} & =e_{j}^{(\alpha, \beta)} b_{j+1}^{(\gamma, \alpha)} b_{j}^{(\gamma, \alpha)}=e_{j}^{(\gamma, \beta)} e_{j+1}^{(\alpha, \gamma)} \\
b_{j-1}^{(\beta, \gamma)} b_{j}^{(\beta, \gamma)} e_{j-1}^{(\alpha, \beta)} & =e_{j}^{(\alpha, \beta)} b_{j-1}^{(\alpha, \gamma)} b_{j}^{(\alpha, \gamma)}=e_{j}^{(\gamma, \beta)} e_{j-1}^{(\alpha, \gamma)}
\end{aligned}
$$

(vi) compatibility relations between projectors and braids:

$$
\begin{gathered}
b_{k}^{ \pm}{ }_{k}^{(\beta, \gamma)} P_{j}^{(\alpha)}=P_{j}^{(\alpha)} b_{k}^{ \pm}{ }_{k}^{(\beta, \gamma)} \quad \text { for } j \neq k, k+1 \\
b_{j}^{ \pm}{ }_{j}^{(\beta, \gamma)} P_{j}^{(\alpha)}=P_{j+1}^{(\alpha)} b_{j}^{ \pm}{ }_{j}^{(\beta, \gamma)}=\delta_{\alpha, \beta} b_{j}^{ \pm}{ }_{j}^{(\beta, \gamma)} \\
b_{j}^{ \pm}{ }_{j}^{(\beta, \gamma)} P_{j+1}^{(\alpha)}=P_{j}^{(\alpha)} b_{j}^{ \pm}{ }_{j}^{(\beta, \gamma)}=\delta_{\alpha, \gamma} b_{j}^{ \pm(\beta, \gamma)}
\end{gathered}
$$

(vii) compatibility relations between projectors and monoids:

$$
\begin{aligned}
& e_{k}^{(\beta, \gamma)} P_{j}^{(\alpha)}=P_{j}^{(\alpha)} e_{k}^{(\beta, \gamma)} \text { for } j \neq k, k+1 \\
& e_{j}^{(\beta, \gamma)} P_{j}^{(\alpha)}=e_{j}^{(\beta, \gamma)} P_{j+1}^{(\alpha)}=\delta_{\alpha, \beta} e_{j}^{(\beta, \gamma)} \\
& P_{j}^{(\alpha)} e_{j}^{(\beta, \gamma)}=P_{j+1}^{(\alpha)} e_{j}^{(\beta, \gamma)}=\delta_{\alpha, \gamma} e_{j}^{(\beta, \gamma)}
\end{aligned}
$$

\subsection{Graphical Interpretation}

The algebra defined above allows a graphical presentation acting on $n+1$ strings which themselves can be thought of as composite objects consisting of $m$ "coloured" strings each. In the pictures below, we are going to represent the "full" ("uncoloured") string by a bold (thick) line whereas strings with a colour are represented by a thin line carrying the colour index. by

Our pictorial view of the generators looks as follows. The projectors $P_{j}^{(\alpha)}$ are represented

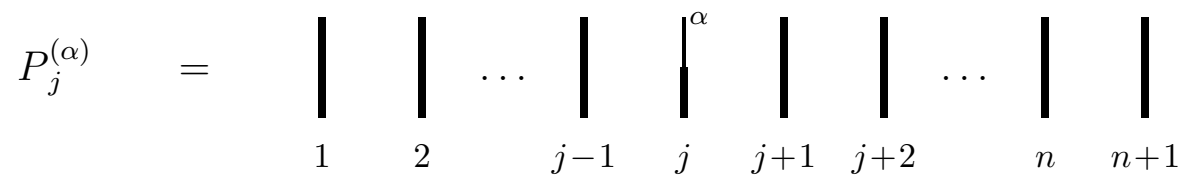


or simpler by

$$
P_{j}^{(\alpha)}=\mid \begin{aligned}
& \mid \\
& 1
\end{aligned}
$$

since we can always multiply with the identity

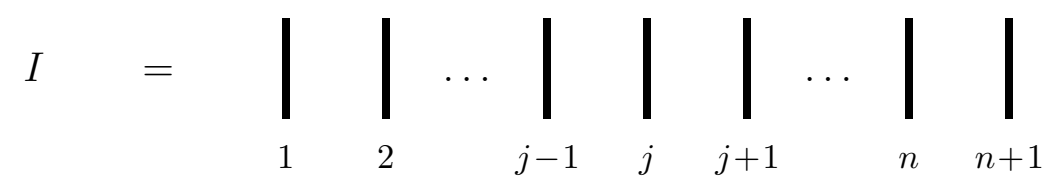

The coloured braids, inverse braids, and monoids correspond to the diagrams

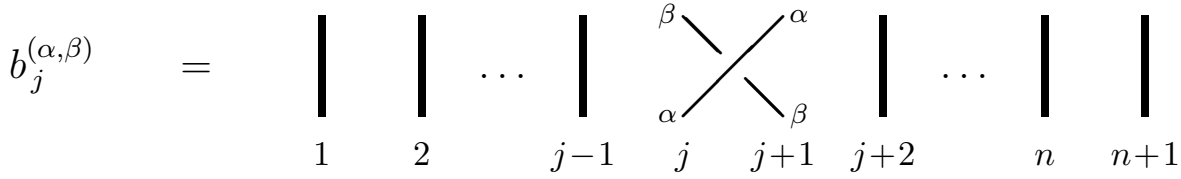

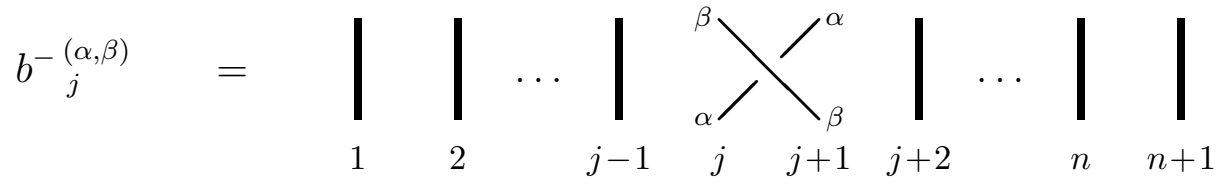

$$
\begin{aligned}
& e_{j}^{(\alpha, \beta)}=\left.\left.\left.\left.\right|_{1} \ldots\right|_{j-1} \overbrace{j}^{\beta} \overbrace{j+1}^{\beta}\right|_{j+2} ^{\beta} \ldots\right|_{n} \mid
\end{aligned}
$$

Multiplication corresponds to concatenation of pictures where we use the convention that the product $A \cdot B$ corresponds to glueing the picture for $A$ above the one for $B$ (hence if one thinks of the generators as operators acting on states (to the right) then the "time-direction" points upwards). Any picture with an incompatible matching of colours is zero. Diagrams which can be transformed into each other by continuous deformations of strings (without affecting the colours of strings, of course) are equivalent. All the defining relations of the multi-colour braid-monoid algebra can be visualized in this way.

To illustrate this, we present pictorial versions of the main defining relations as examples. The projector relations (2.1) and the compatibility relations (2.6) and (2.7) follow from the requirement of matching colours alone. Also, the commutativity of operators acting on different strings is represented in the diagrams in an obvious way. For the remaining relations, the corresponding pictures are given in the sequel. 
The braid relations (2.2) give rise to the following diagrams
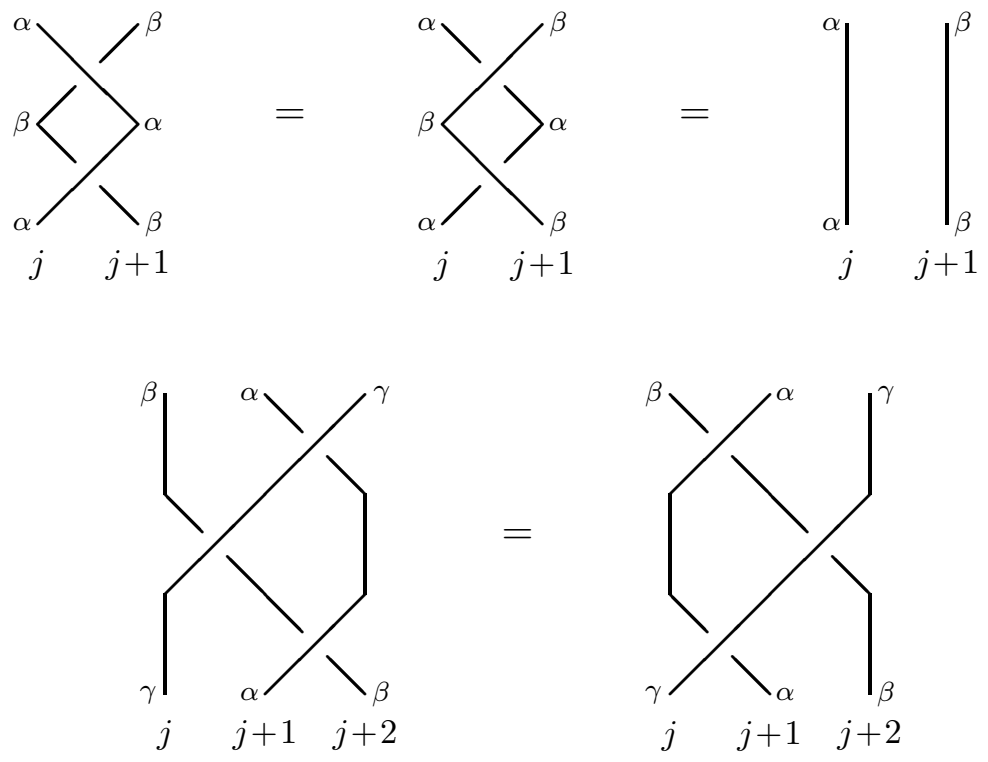

where here and in what follows we only show the relevant part.

The multi-colour Temperley-Lieb relations (2.3) are
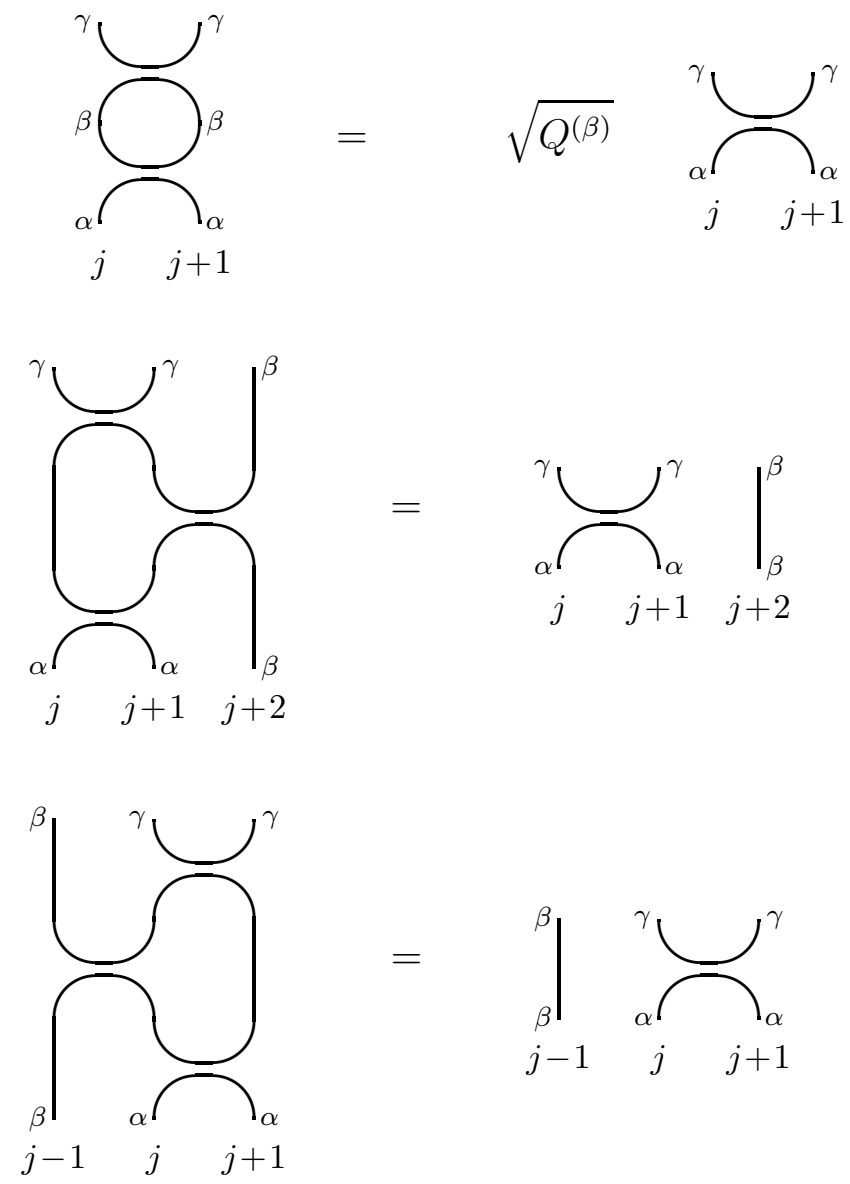
which means that a closed loop of colour $\alpha$ in a diagram can be replaced by the diagram without the loop multiplied by a factor $\sqrt{Q^{(\alpha)}}$.

The "twist" relations (2.4) have the following pictorial interpretation
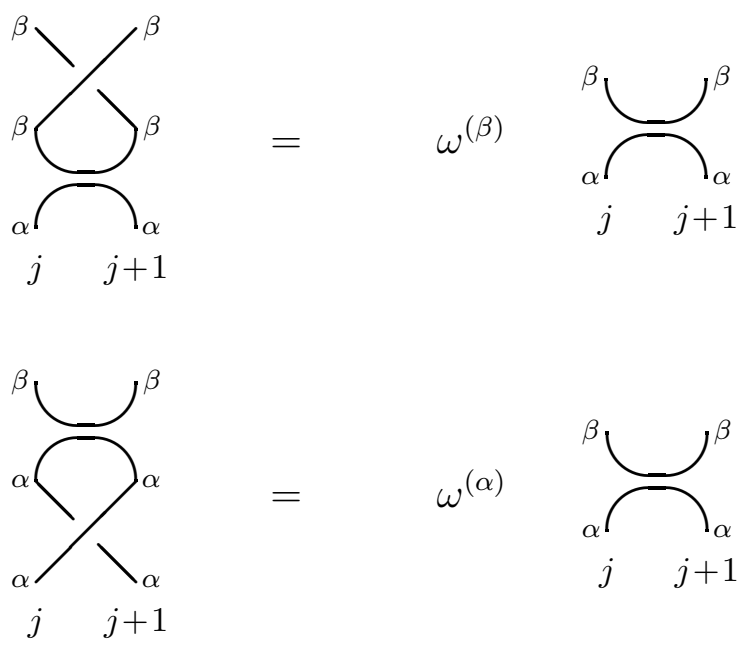

i.e., undoing a "twist" in a string of colour $\alpha$ produces a factor $\omega^{(\alpha)}$.

Finally, the braid-monoid relations (2.5) look as follows
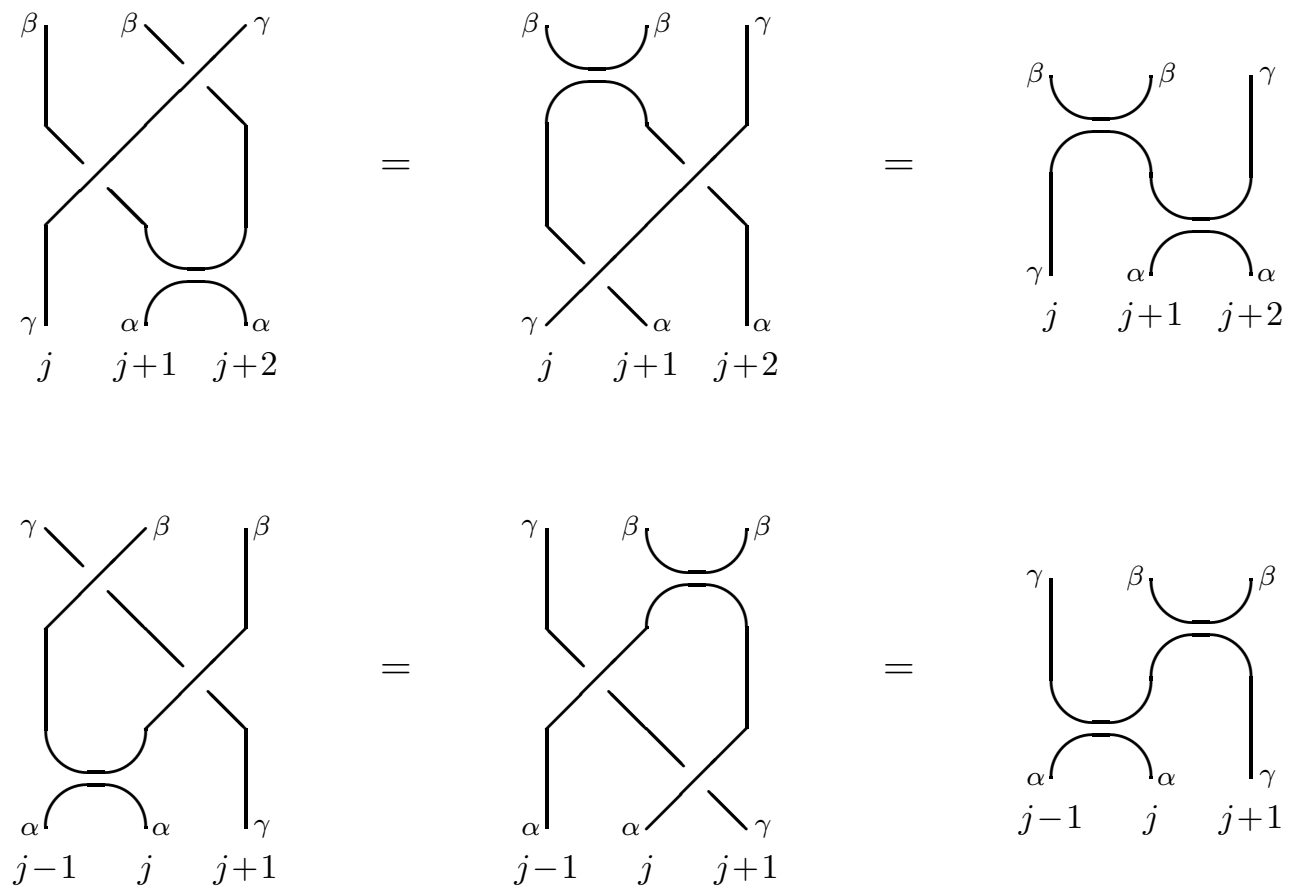

This completes the list of defining relations. The pictorial presentation is useful since it allows to simplify products of operators in a fast and easy way. More importantly, however, it shows that there is a close connection to the theory of coloured knots and links and it should be possible to derive invariants for the coloured objects in the same way one obtains invariants for knots and links from the usual braid-monoid algebra (see e.g. [8] and [18]). 


\subsection{Generalized Crossing Symmetry}

Before we commence to investigate representations of the multi-colour algebra, let us say a few words on a generalization of the so-called crossing symmetry which is actually a built-in feature of the algebra. The notion of crossing symmetry originates in scattering theory and was introduced in the context of statistical mechanics models via the close relation of exactly solvable two-dimensional statistical models and completely integrable quantum systems in one dimension (where the Yang-Baxter equations guarantee the factorization of S-matrices, see e.g. [8]), compare the discussion of crossing symmetry in solvable models in sec. 4.1 below. We do not attempt to formulate crossing in full generality but we rather present some simple examples which clarify our notion of crossing. Since the colours of strings are not altered by crossing we neglect all the colour indices of the strings for the moment. However, one should bear in mind that each string is meant to have a definite colour in the pictures below (or that one anyhow looks at the one-colour case). This restriction is necessary here since we do not have an interpretation for crossing of the projectors.

To explain what we mean by generalized crossing, we use the graphical interpretation of the algebra. Essentially, if one has any (sub-) diagram (symbolized by a rectangular box in the figure below) with $\ell$ in- and outgoing (coloured) strings,

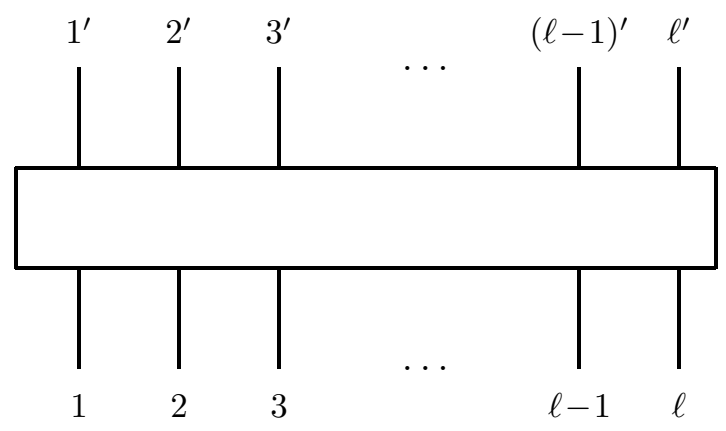

applying one generalized crossing step ("crossing generator") means that one changes the interpretation of two strings as follows:
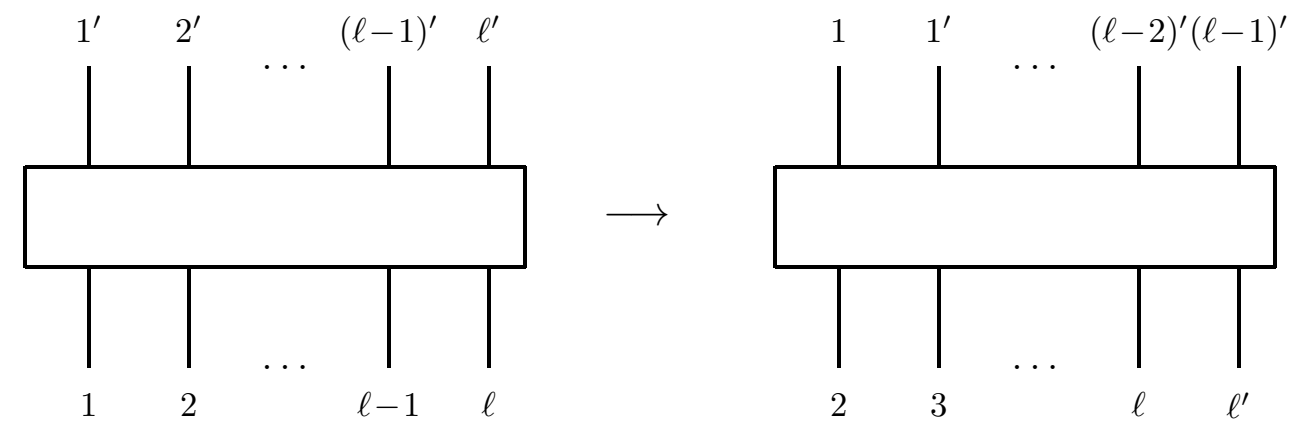

i.e., one in-string is converted into an out-string and vice versa. Note that the relative order of the strings is maintained. The diagrams that one obtains this way can again be interpreted as representing products of generators in our algebra (no projectors), although one might 
have to add additional strings as shown in the example below.
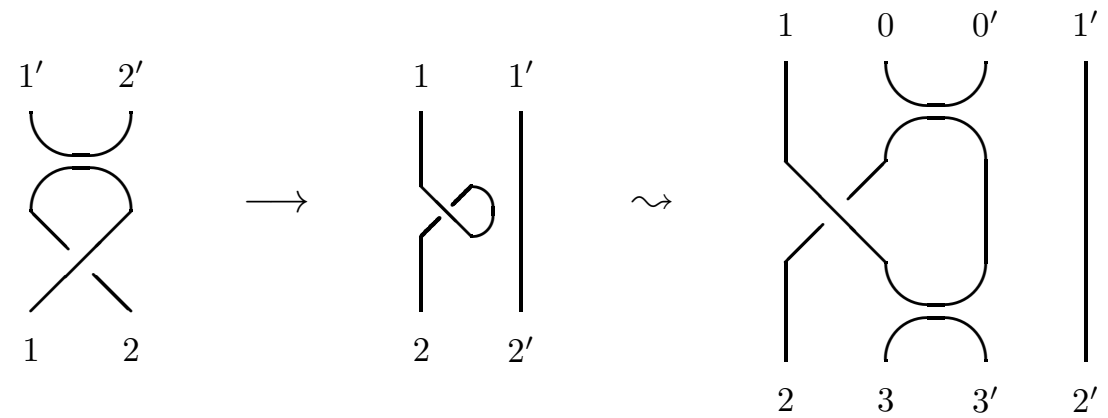

The labels $\left(0,0^{\prime}\right)$ and $\left(3,3^{\prime}\right)$ for the additional lines are motivated by the fact that the last diagram can also be obtained from

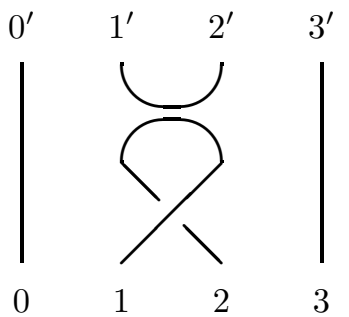

by two generalized crossing steps.

As already mentioned above, we cannot give a natural interpretation of crossing for the projectors since they only act in one direction. Nevertheless, we certainly can "cross" straight lines that have a definite colour from the beginning (i.e., one thinks of the operators acting on coloured strings already which means that the projectors just act as the identity) to obtain coloured monoids. For the remaining generators, one has

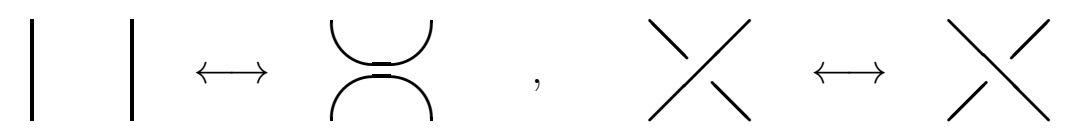

i.e., crossing maps the identity to the monoid and the braid to its inverse. Thus, by crossing twice, one gets back the diagram one started with. Note that this is only true if one has only one colour, otherwise

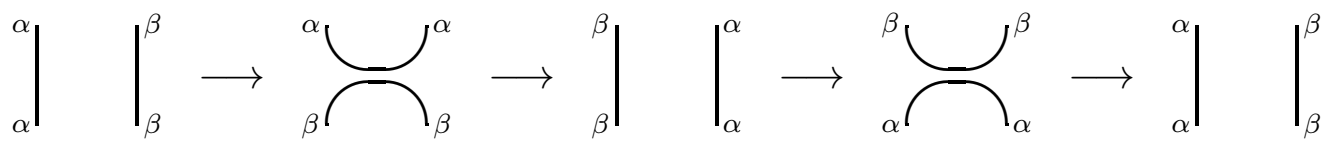

whereas for the coloured braid one still has

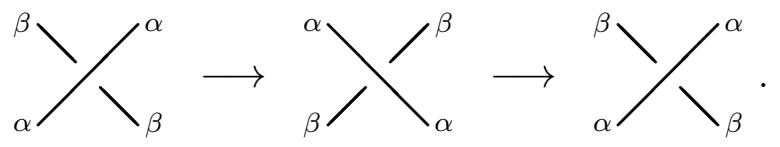


Of course, one always comes back to the operator one started with after four generalized crossing steps (for $\ell=2$, in general one needs $2 \ell$ steps).

Crossing symmetry now means that if one has a relation in the algebra and performs a generalized crossing transformation on both sides of the equation, one obtains - possibly after adding strings in order to be able to interpret the resulting diagrams as representing products of generators of the algebra - another valid equation in the algebra. Of course, adding strings has to be done the same way on both sides of the equation.

To conclude our excursion with an instructive example, we show how one of the braidmonoid relations of eq. (2.5) is crossing-related to the simple fact that a braid multiplied with its inverse yields the identity. In the coloured case, it looks as follows

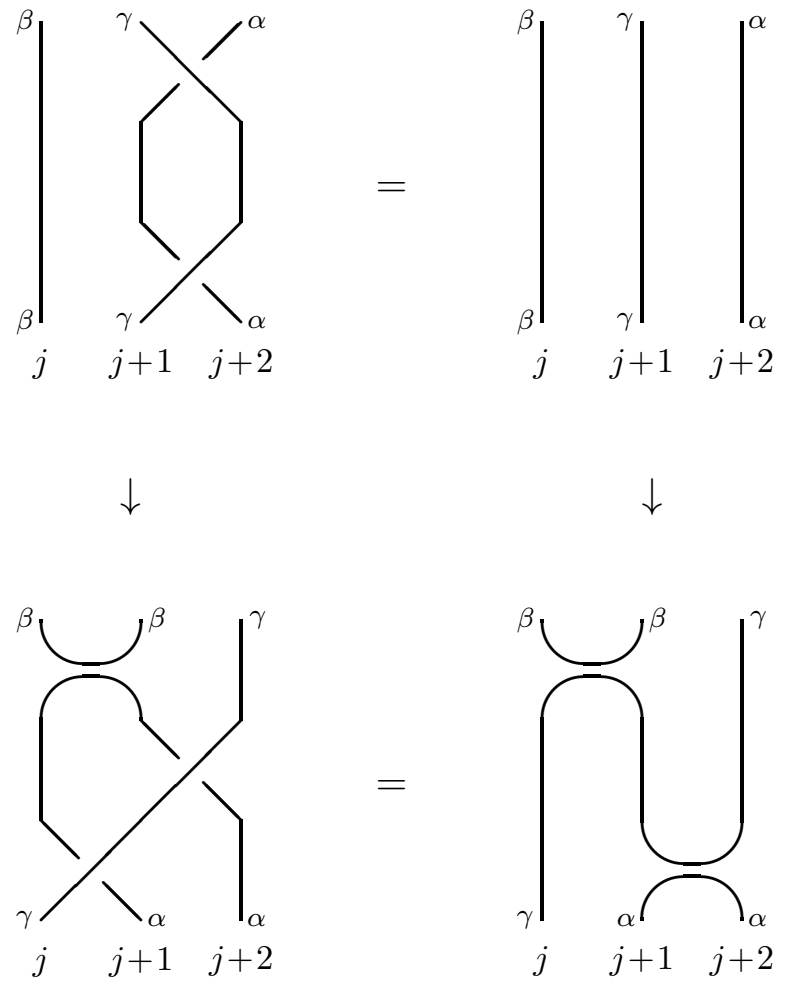

This also means that one could significantly reduce the number of defining relations of the multi-colour algebra by imposing crossing symmetry. We chose not to do so since it is not easy to implement crossing algebraically. That also explains why we used the diagrammatic interpretation of the algebra in the above discussion.

\section{Representations}

In the context of exactly solvable models, we are interested in representations of the multicolour braid-monoid algebra where the central elements $\sqrt{Q^{(\alpha)}}$ and $\omega^{(\alpha)}(1 \leq \alpha \leq m)$ are represented by numbers and where the following equations hold

$$
f^{(\alpha)}\left(b_{j}^{(\alpha, \alpha)}\right) p_{j}^{(\alpha, \alpha)}=0
$$




$$
\begin{aligned}
f^{(\alpha, \beta)}\left(b_{j}^{(\beta, \alpha)} b_{j}^{(\alpha, \beta)}\right) p_{j}^{(\alpha, \beta)} & =0 \\
g^{(\alpha)}\left(b_{j}^{(\alpha, \alpha)}\right) p_{j}^{(\alpha, \alpha)} & =e_{j}^{(\alpha, \alpha)}
\end{aligned}
$$

where $f^{(\alpha)}(z), f^{(\alpha, \beta)}(z)$, and $g^{(\alpha)}(z)(1 \leq \alpha, \beta \leq m)$ are polynomials in $z$ and where we introduced "two-site projectors" $p_{j}^{(\alpha, \beta)}$ by

$$
p_{j}^{(\alpha, \beta)}=P_{j}^{(\alpha)} P_{j+1}^{(\beta)}
$$

as a convenient abbreviation円. We regard the above relations (3.1)-(3.3) as properties of the representations rather than defining relations of the algebra since the actual numbers and polynomials are model-dependent quantities.

The following remarks are in order:

(i) For the one-colour case $(m=1)$ the algebra defined above reduces to the well-known braid-monoid algebra (see e.g. [8])

(ii) From any representation $\varrho$ of the one-colour algebra which acts in an $(n+1)$-fold tensor product space

$$
\varrho: \bigotimes_{k=1}^{n+1} \mathcal{V}_{k} \longrightarrow \bigotimes_{k=1}^{n+1} \mathcal{V}_{k}
$$

one can obtain representations $\varrho^{(m)}$ of the $m$-colour case as follows: As representation space at site $j$ choose the $m$-fold direct sum $V_{j}=\mathcal{V}_{j}^{(1)} \oplus \mathcal{V}_{j}^{(2)} \oplus \ldots \oplus \mathcal{V}_{j}^{(m)}$ of the corresponding space of the one-colour representation $\mathcal{V}_{j}^{(\alpha)} \cong \mathcal{V}_{j}$ and represent the operators $P_{j}^{(\alpha)}(1 \leq \alpha \leq m)$ by the orthogonal projectors onto the $m$ subspaces $\mathcal{V}_{j}^{(\alpha)}$ at site $j$. From the compatibility relations (2.6) and (2.7), it follows immediately that $b_{j}^{ \pm} \underset{j}{(\alpha, \beta)}$ and $e_{j}^{(\alpha, \beta)}$ can act non-trivially between certain subspaces only. Defining

$$
\begin{aligned}
b_{j}^{ \pm}{ }_{j}^{(\alpha, \beta)} \mid \bigotimes_{k} v_{k}^{\left(\gamma_{k}\right)} \longrightarrow \bigotimes_{k} \mathcal{V}_{k}^{\left(\delta_{k}\right)} & =\left(\prod_{k \neq j, j+1} \delta_{\gamma_{k}, \delta_{k}}\right) \delta_{\alpha, \gamma_{j}, \delta_{j+1}} \delta_{\beta, \gamma_{j+1}, \delta_{j}} b_{j}^{ \pm 1} \\
e_{j}^{(\alpha, \beta)} \mid \bigotimes_{k} v_{k}^{\left(\gamma_{k}\right)} \longrightarrow \bigotimes_{k} \mathcal{V}_{k}^{\left(\delta_{k}\right)} & =\left(\prod_{k \neq j, j+1} \delta_{\gamma_{k}, \delta_{k}}\right) \delta_{\alpha, \gamma_{j}, \gamma_{j+1}} \delta_{\beta, \delta_{j}, \delta_{j+1}} e_{j}
\end{aligned}
$$

where the products over $k$ run from 1 to $n+1$ and

$$
\delta_{a_{1}, a_{2}, \ldots, a_{l}}=\prod_{k=2}^{l} \delta_{a_{1}, a_{k}}
$$

one obtains a representation of the $m$-colour braid-monoid algebra with $\sqrt{Q^{(\alpha)}}=\sqrt{Q}$, $\omega^{(\alpha)}=\omega, f^{(\alpha)}(z)=f(z), f^{(\alpha, \beta)}(z)=f(z) f(-z)$, and $g^{(\alpha)}(z)=g(z)$ for all $\alpha, \beta=$

\footnotetext{
${ }^{1}$ Of course, it is possible to use $p_{j}^{(\alpha, \beta)}$ instead $P_{j}^{(\alpha)}$ to define the algebra from the very beginning. We choose to do otherwise since in our view, the $P_{j}^{(\alpha)}$ are the more basic objects.
} 
$1, \ldots, m$. Here, the quantities without colour indices $\alpha$ or $\beta$ refer to the one-colour case. In particular, this shows that there exist representations of the $m$-colour algebra for any $m$.

(iii) Conversely, starting from a representation of the $m$-colour algebra $(m>1)$, one can recover part of the one-colour relations for the "full" or "uncoloured" Temperley-Lieb and braid operators obtained by summing over all colours. To be more precise, the operators $B_{j}^{ \pm}$defined by

$$
B_{j}^{ \pm}=\sum_{\alpha, \beta=1}^{m}\left(c^{(\alpha, \beta)}\right)^{ \pm 1} b_{j}^{ \pm}{ }_{j}^{(\alpha, \beta)}
$$

with $c^{(\alpha, \beta)} \in \mathbb{C} \backslash\{0\}$ fulfill the braid algebra relations $(2.2)$ and the operators $E_{j}$ defined by

$$
E_{j}=\sum_{\alpha, \beta=1}^{m}\left(\frac{c^{(\beta)}}{c^{(\alpha)}}\right) e_{j}^{(\alpha, \beta)}
$$

$\left(c^{(\alpha)} \in \mathbb{C} \backslash\{0\}\right)$ generate the Temperley-Lieb algebra $(2.3)$ with $\sqrt{Q}=\sum_{\alpha=1}^{m} \sqrt{Q^{(\alpha)}}$. However, the relations (2.4) and (2.5) between these two types of operators are in general not satisfied by $B_{j}^{ \pm}$and $E_{j}$ as they are defined in Eqs. (3.9) and (3.10) above. In particular, the algebra generated by the "full" braids and monoids in general becomes non-abelian at one site $j$. The relations (2.5) hold if and only if $\left(c^{(\alpha, \beta)}\right)^{2}=1$ for all $\alpha, \beta=1, \ldots, m$. Eq. (2.4) is fulfilled with twist $\omega$ if and only if $c^{(\alpha, \alpha)} \omega^{(\alpha)}=\omega$ for all $\alpha=1, \ldots, m$. Hence the full set of relations can only be recovered if all the twists $\omega^{(\alpha)}$ coincide up to a sign. It should be added that whereas one again has polynomial equations in the "full" braid" $B_{j}$ (3.1) one obviously cannot write the "full" TemperleyLieb operator $E_{j}$ as a polynomial in the braid $B_{j}(3.3)$ (unless all $e_{j}^{(\alpha, \beta)}$ with $\alpha \neq \beta$ are represented by zero matrices).

In what follows, we construct three classes of representations for the $m$-colour braidmonoid algebra. These representations are related to exactly solvable lattice models of statistical mechanics as we are going to show in sec. 4 .

\subsection{Vertex-Type Representations}

Representations of the (one-colour) braid-monoid algebra which are linked to vertex models (see eg. 81) are of the type (3.5), i.e., they act in a tensor product space. Therefore, eqs. (3.6) and (3.7) give $m$-colour generalizations of this kind of representations.

Here, we construct a different multi-colour generalization of the representation of the one-colour algebra related to the 6-vertex model (see eg. 8]) which is the simplest (nontrivial) vertex model related to the affine Lie algebra $A_{1}^{(1)}$ [19. The representation of the $m$-colour braid-monoid algebra acts in the space $\left(\mathcal{V}^{m}\right)^{\otimes(n+1)}$ with $\mathcal{V} \cong \mathbb{C}^{2}$ according to the two possible states (arrows) of the 6 -vertex model. The operators $P_{j}^{(\alpha)}, b_{j}^{ \pm} \underset{j}{(\alpha, \beta)}$, and $e_{j}^{(\alpha, \beta)}$

\footnotetext{
${ }^{2}$ This follows from the fact that there are only finitely many independent products of coloured braids which enter in the expressions for the powers of the "full" braid $B_{j}$.
} 
act as the identity in all but the space $\left(\mathcal{V}^{m}\right)_{j}\left(\right.$ for $\left.P_{j}^{(\alpha)}\right)$ respective $\left(\mathcal{V}^{m}\right)_{k}$ with $k=j, j+1$ $\left(\right.$ for $b_{j}^{ \pm}{ }_{j}^{(\alpha, \beta)}$ and $\left.e_{j}^{(\alpha, \beta)}\right)$, i.e.,

$$
\begin{aligned}
P_{j}^{(\alpha)} & =\left(\bigotimes_{k=1}^{j-1} 1\right) \otimes P^{(\alpha)} \otimes\left(\bigotimes_{k=j+1}^{n+1} 1\right) \\
b_{j}^{ \pm(\alpha, \beta)} & =\left(\bigotimes_{k=1}^{j-1} 1\right) \otimes b^{ \pm(\alpha, \beta)} \otimes\left(\bigotimes_{k=j+2}^{n+1} 1\right) \\
e_{j}^{(\alpha, \beta)} & =\left(\bigotimes_{k=1}^{j-1} 1\right) \otimes e^{(\alpha, \beta)} \otimes\left(\bigotimes_{k=j+2}^{n+1} 1\right) .
\end{aligned}
$$

Here, $P^{(\alpha)}: \mathcal{V}^{m} \longrightarrow \mathcal{V}^{m}$ acts as a projector onto colour $\alpha$

$$
P^{(\alpha)}=\left(\bigoplus_{\beta=1}^{\alpha-1} 0\right) \oplus 1 \oplus\left(\bigoplus_{\beta=\alpha+1}^{m} 0\right)
$$

whereas $b^{ \pm(\alpha, \beta)}$ and $e^{(\alpha, \beta)}$ both map $\mathcal{V}^{m} \otimes \mathcal{V}^{m} \longrightarrow \mathcal{V}^{m} \otimes \mathcal{V}^{m}$. As above (see eqs. (3.6) and (3.7)), the compatibility relations (2.6) and (2.7) imply that $b^{ \pm(\alpha, \beta)}$ and $e^{(\alpha, \beta)}$ can have non-zero matrix elements in certain subspaces only. In these subspaces, they are given by $4 \times 4$ matrices with elements

$$
\begin{aligned}
&\left(b^{ \pm(\alpha, \alpha)} \mid \mathcal{V}^{(\alpha) \otimes \mathcal{V}^{(\alpha)} \rightarrow \mathcal{V}^{(\alpha)} \otimes \mathcal{V}^{(\alpha)}}\right)_{(s, t),\left(s^{\prime}, t^{\prime}\right)}=\left(k^{(\alpha)}\right)^{ \pm 1}\left(\delta_{s, t^{\prime}} \delta_{s^{\prime}, t}-\right. \\
&\left(b^{ \pm(\alpha, \beta)} \mid \mathcal{V}^{(\alpha)} \otimes \mathcal{V}^{(\beta)} \rightarrow \mathcal{V}^{(\beta)} \otimes \mathcal{V}^{(\alpha)}\right)_{(s, t),\left(s^{\prime}, t^{\prime}\right)}^{ \pm 1-\left(s+s^{\prime}\right) / 2} \delta_{s,-t} \delta_{s^{\prime},-t^{\prime}}=\delta_{s, t^{\prime}} \delta_{s^{\prime}, t} \quad \text { for } \alpha \neq \beta \\
&\left(e^{(\alpha, \beta)} \mid \mathcal{V}^{(\alpha) \otimes \mathcal{V}^{(\alpha)} \rightarrow \mathcal{V}^{(\beta)} \otimes \mathcal{V}^{(\beta)}}\right)_{(s, t),\left(s^{\prime}, t^{\prime}\right)}=\left(x^{(\alpha)}\right)^{-s}\left(x^{(\beta)}\right)^{-s^{\prime}} \delta_{s,-t} \delta_{s^{\prime},-t^{\prime}}
\end{aligned}
$$

where $s, t, s^{\prime}, t^{\prime}= \pm 1$. Here, $x^{(\alpha)}=\exp \left(i \lambda^{(\alpha)}\right)$ and $k^{(\alpha)}=-i \exp \left(-i \lambda^{(\alpha)} / 2\right)=1 /\left(i \sqrt{x^{(\alpha)}}\right)$, and $\lambda^{(\alpha)}$ are arbitrary (real or complex) numbers which one can choose independently for any colour $\alpha$.

The above representation (which we denote by $(6-\mathrm{V}, 6-\mathrm{V}, \ldots, 6-\mathrm{V}))$ is characterized by

$$
\begin{aligned}
\sqrt{Q^{(\alpha)}} & =2 \cos \left(\lambda^{(\alpha)}\right)=x^{(\alpha)}+1 / x^{(\alpha)} \\
\omega^{(\alpha)} & =-\left(k^{(\alpha)}\right)^{-3} \\
f^{(\alpha)}(z) & =\left(z-\omega^{(\alpha)}\right)\left(z-k^{(\alpha)}\right)
\end{aligned}
$$




$$
\begin{aligned}
f^{(\alpha, \beta)}(z) & =z-1 \quad(\alpha \neq \beta) \\
g^{(\alpha)}(z) & =k^{(\alpha)}\left(z-k^{(\alpha)}\right) .
\end{aligned}
$$

Note that even in the case of $m$ identical values of $\lambda^{(\alpha)} \equiv \lambda$ this representation is in general different from the representation given in eqs. (3.6) and (3.7). This is obvious since $f^{(\alpha, \beta)}(z)=z-1$ here whereas $f^{(\alpha, \beta)}(z)=\left(z^{2}-\omega^{2}\right)\left(z^{2}-k^{2}\right)$ in the other case.

\subsection{Representations labelled by Graphs}

Consider $m$ (connected) graphs $\mathcal{G}^{(\alpha)}, 1 \leq \alpha \leq m$, with $L^{(\alpha)}$ nodes (enumerated by $a^{(\alpha)}=$ $\left.1, \ldots, L^{(\alpha)}\right)$ where any pair of nodes is connected by at most one line (bond). Connectivity is not really a restriction here, in fact one can always think of the connected components of one graph as separate graphs. Note that we do not have to assume that the graphs are simple, i.e. that each line connects two distinct nodes. In what follows, we denote by $\mathcal{N}^{(\alpha)}=\left\{1, \ldots, L^{(\alpha)}\right\}$ the set of all nodes of $\mathcal{G}^{(\alpha)}$.

To each graph $\mathcal{G}^{(\alpha)}$ we associate an adjacency matrix $\mathcal{A}^{(\alpha)}$. This $L^{(\alpha)} \times L^{(\alpha)}$ matrix has elements

$$
\mathcal{A}_{a^{(\alpha)}, b^{(\alpha)}}^{(\alpha)}=\mathcal{A}_{b^{(\alpha)}, a^{(\alpha)}}^{(\alpha)}= \begin{cases}1 & \text { if } a^{(\alpha)} \text { and } b^{(\alpha)} \text { are adjacent in } \mathcal{G}^{(\alpha)} \\ 0 & \text { otherwise }\end{cases}
$$

where $a^{(\alpha)}, b^{(\alpha)} \in\left\{1, \ldots, L^{(\alpha)}\right\}$ are adjacent if the nodes $a^{(\alpha)}$ and $b^{(\alpha)}$ in $\mathcal{G}^{(\alpha)}$ are connected by a bond. In other words, the adjacency matrices considered here are characterized by being symmetric matrices with all entries 0 or 1 and by vanishing elements on the diagonal in case the corresponding graphs are simple. We denote by

$$
\begin{aligned}
\Lambda^{(\alpha)} & =2 \cos \left(\lambda^{(\alpha)}\right)=x^{(\alpha)}+1 / x^{(\alpha)} \\
& =-2 \cos \left(4 \bar{\lambda}^{(\alpha)}\right)=-\left(\left(y^{(\alpha)}\right)^{4}+\left(y^{(\alpha)}\right)^{-4}\right)
\end{aligned}
$$

the Perron-Frobenius eigenvalue of $\mathcal{A}^{(\alpha)}$ and by $S_{j}^{(\alpha)}\left(1 \leq j \leq L^{(\alpha)}\right)$ the elements of the corresponding eigenvector, i.e.,

$$
\sum_{i=1}^{L^{(\alpha)}} \mathcal{A}_{i, j}^{(\alpha)} S_{j}^{(\alpha)}=\Lambda^{(\alpha)} S_{i}^{(\alpha)}
$$

The two different parametrizations in eqs. (3.20) and (3.21) will prove useful below. Note that whereas for fixed $\Lambda^{(\alpha)}$ there is only one value for $\lambda^{(\alpha)}$ (modulo $2 \pi$ and up to a sign which is irrelevant here) there are in general four different values for $\bar{\lambda}^{(\alpha)}$, namely $\bar{\lambda}^{(\alpha)}=\left(\lambda^{(\alpha)} \pm \pi\right) / 4$ and $\bar{\lambda}^{(\alpha)}=\left(\lambda^{(\alpha)} \pm 3 \pi\right) / 4$.

Consider the cartesian product $\mathcal{G}=\mathcal{G}^{(1)} \times \mathcal{G}^{(2)} \times \ldots \times \mathcal{G}^{(m)}$ of the $m$ graphs. It has $L=\prod_{\alpha=1}^{m} L^{(\alpha)}$ nodes labelled by $\mathcal{N}=\mathcal{N}^{(1)} \times \mathcal{N}^{(2)} \times \ldots \times \mathcal{N}^{(m)}$ and hence the corresponding adjacency matrix $A$ is an $L \times L$ matrix given by

$$
A=\mathcal{A}^{(1)} \times \mathcal{A}^{(2)} \times \ldots \times \mathcal{A}^{(m)}=\sum_{\alpha=1}^{m} A^{(\alpha)}
$$


where $A^{(\alpha)}$ is the $L \times L$ matrix with elements

$$
A_{a, b}^{(\alpha)}=\left(\prod_{\beta \neq \alpha} \delta_{a^{(\beta)}, b^{(\beta)}}\right) \mathcal{A}_{a^{(\alpha)}, b^{(\alpha)}}^{(\alpha)}
$$

and we use $m$-tupels $a=\left(a^{(1)}, a^{(2)}, \ldots, a^{(m)}\right) \in \mathcal{N}$ to enumerate the nodes of $\mathcal{G}$ (which we also call "states" or "heights" in reminiscence of the role they play in the solvable models).

The basis $N_{n}$ of our representation space for the $(n+1)$-string $m$-colour braid-monoid algebra is now given by all allowed $(n+1)$-step paths in the graph $\mathcal{G}$, that is by the following set

$$
N_{n}=\left\{\boldsymbol{a}=\left(a_{0}, a_{1}, \ldots, a_{n}, a_{n+1}\right) \mid a_{j} \in \mathcal{N} \text { and } \prod_{j=1}^{n+1} A_{a_{j-1}, a_{j}}=1\right\}
$$

which forms a subset of the $(n+2)$-fold cartesian product of the set $\mathcal{N}$. Then the following equations define a matrix representation of the $m$-colour algebra on the space spanned by $N_{n}$ :

$$
\begin{aligned}
& \left(P_{j}^{(\alpha)}\right)_{\boldsymbol{a}, \boldsymbol{b}}=\left(\prod_{k=0}^{n+1} \delta_{a_{k}, b_{k}}\right) A_{a_{j-1}, a_{j}}^{(\alpha)}
\end{aligned}
$$

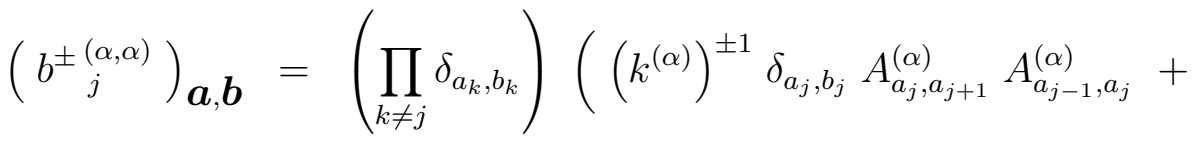

$$
\begin{aligned}
& \left.\left(k^{(\alpha)}\right)^{\mp 1} \sqrt{\frac{S_{a_{j}} S_{b_{j}}}{S_{a_{j-1}}^{2}}} \delta_{a_{j-1}, a_{j+1}} A_{a_{j}, a_{j+1}}^{(\alpha)} A_{a_{j-1}, b_{j}}^{(\alpha)}\right) \\
& \left(b_{j}^{ \pm}(\alpha, \beta)\right)_{\boldsymbol{a}, \boldsymbol{b}}=\left(\prod_{k \neq j} \delta_{a_{k}, b_{k}}\right) A_{a_{j-1}, b_{j}}^{(\alpha)} A_{b_{j}, a_{j+1}}^{(\beta)} A_{a_{j}, a_{j+1}}^{(\alpha)} A_{a_{j-1}, a_{j}}^{(\beta)} \quad(\alpha \neq \beta) \\
& \left(e_{j}^{(\alpha, \beta)}\right)_{\boldsymbol{a}, \boldsymbol{b}}=\left(\prod_{k \neq j} \delta_{a_{k}, b_{k}}\right) \delta_{a_{j-1}, a_{j+1}} \sqrt{\frac{S_{a_{j} S_{b_{j}}}}{S_{a_{j-1}}^{2}}} A_{a_{j-1}, b_{j}}^{(\alpha)} A_{a_{j}, a_{j+1}}^{(\beta)}
\end{aligned}
$$

where $\boldsymbol{a}=\left(a_{0}, a_{1}, \ldots, a_{n+1}\right), \boldsymbol{b}=\left(b_{0}, b_{1}, \ldots, b_{n+1}\right)$, and where

$$
k^{(\alpha)}=\frac{1}{i \sqrt{x^{(\alpha)}}}=-\left(y^{(\alpha)}\right)^{-2}
$$

fixes the scale of the braids $b_{j}^{(\alpha, \alpha)}$ such that eqs. (2.5) are satisfied ${ }^{3}$.

These representations are characterized by (cf. eqs. (3.1)-(3.3))

$$
\begin{aligned}
\sqrt{Q^{(\alpha)}} & =\Lambda^{(\alpha)} \\
\omega^{(\alpha)} & =-\left(k^{(\alpha)}\right)^{-3}
\end{aligned}
$$

\footnotetext{
${ }^{3}$ Note that eqs. (2.5) fix the scale of the braids up to a sign.
} 


$$
\begin{aligned}
f^{(\alpha)}(z) & =\left(z-\omega^{(\alpha)}\right)\left(z-k^{(\alpha)}\right) \\
f^{(\alpha, \beta)}(z) & =z-1 \quad(\alpha \neq \beta) \\
g^{(\alpha)}(z) & =k^{(\alpha)}\left(z-k^{(\alpha)}\right)
\end{aligned}
$$

For the one-colour case, the representation defined through eqs. (3.26)-(3.30) reduces to the representation related to the Temperley-Lieb interaction models [3, 20, 21]. These are critical IRF models [22] if the underlying graph is a Dynkin diagram of a simply-laced Lie algebra or of the corresponding affine algebra [21]. They include e.g. the critical A-D-E lattice models of Pasquier [23, 24, 25] (which in turn include the ABF or RSOS (restricted solid-on-solid) models [26]) and the CSOS (cyclic solid-on-solid) models [27, 28, 29]. For this reason, the present class of representations will frequently be referred to as "RSOS representation" below.

The apparent agreement between the polynomial relations satisfied by the vertex-type representation (3.18) and the present case (3.31) is not coincidental. If one considers the representation labelled by $\left(\mathrm{A}_{L^{(1)}}^{(1)}, \mathrm{A}_{L^{(2)}}^{(1)}, \ldots, \mathrm{A}_{L^{(m)}}^{(1)}\right)$ and performs the limits $L^{(\alpha)} \longrightarrow \infty$ with $\lambda^{(\alpha)}=\pi s^{(\alpha)} / L^{(\alpha)}\left(0<s^{(\alpha)}<L^{(\alpha)}, s^{(\alpha)}\right.$ and $L^{(\alpha)}$ coprime $)$ fixed , one obtains an infinite dimensional representation of the $m$-colour algebra which nevertheless is related to the vertex representation (3.11)-(3.17) of the $(n+1)$-string $m$-colour braid monoid algebra with the corresponding values of $\lambda^{(\alpha)}$. In the limit, the dependence on the actual heights vanishes all that matters are differences of heights. Since for any colour there are only two possibilities (height either increasing or decreasing by one), one can go over to a description with only two states which is the corresponding vertex-type representation. This is nothing but the usual SOS (solid-on-solid) - vertex model correspondence (Wu-Kadanoff-Wegner transformation, see [30, 31]) which will also be reflected in our examples of solvable models below.

\subsection{Mixed Representations}

As "mixed" representations $\left(6 \mathrm{~V}, 6 \mathrm{~V}, \ldots, 6 \mathrm{~V}, \mathcal{G}^{(1)}, \mathcal{G}^{(2)}, \ldots, \mathcal{G}^{\left(m_{R}\right)}\right)$ we denote representations of the $m$-colour braid-monoid algebra in which $m_{V}$ colours appear in the vertex-type representation and the other $m_{R}=m-m_{V}$ in the RSOS representation labelled by the $m_{R}$ graphs $\mathcal{G}^{(1)}, \mathcal{G}^{(2)}, \ldots, \mathcal{G}^{\left(m_{R}\right)}$. For simplicity, we assume that among those all graphs are simple.

Due to the mixture of degrees of freedom on vertices and on edges it is more cumbersome to describe the representation space in the mixed case. We use the notation of sec. 3.2 for the colours labelled by the $m_{R}$ simple graphs. For the vertex part, we use the set

$$
\mathcal{M}=\left\{s=\left(s^{(1)}, s^{(2)}, \ldots, s^{\left(m_{V}\right)}\right) \mid s^{(\alpha)} \in\{-1,0,1\} \text { and }|s|=\sum_{\alpha=1}^{m_{V}}\left|s^{(\alpha)}\right| \leq 1\right\}
$$

which gives all possible vertex states on any edge of the lattice. Here, 0 stands for no arrow and \pm 1 for an arrow that points upwards (downwards) or to the right (left), respectively. The second condition guarantees that there is at most one coloured arrow on any edge.

\footnotetext{
${ }^{4}$ Note that in order to do this one has to use other eigenvalues and eigenvectors of the adjacency matrices apart from the Perron-Frobenius one. Of course, one can use any eigenvalue and eigenvector, the only benefit of using the Perron-Frobenius vector being that it has real positive entries only.
} 
The representation space $N_{n}$ now consists of all paths of the form

$$
\begin{gathered}
N_{n}=\left\{\boldsymbol{a}=\left(a_{0}, s_{1}, a_{1}, s_{2}, a_{2}, \ldots, a_{n}, s_{n+1}, a_{n+1}\right) \mid a_{j} \in \mathcal{N}, s_{j} \in \mathcal{M}\right. \text { and } \\
\left.\left(A_{a_{j-1}, a_{j}}=1,\left|s_{j}\right|=0\right) \text { or }\left(a_{j-1}=a_{j},\left|s_{j}\right|=1\right)\right\}
\end{gathered}
$$

that is, whenever heights on neighbouring vertices differ by one step on the graph $\mathcal{G}$, there is no arrow on the bond. On the other hand, if there is a coloured arrow on any bond, then it has to join two vertices with equal heights. Other configurations are not allowed.

Now, let us turn to the matrix representations of the generators. In case they only involve colours of vertex-type, the other degrees of freedom play no role and the matrices are essentially given by eqs. (3.11)-(3.17). On the other hand, if only colours of RSOS-type are involved, the vertex degrees of freedom do not contribute and the relevant parts of the matrices are given by eqs. (3.26)-(3.29). Hence, all that remains are the two-colour braids or monoids where one colour corresponds to a vertex and the other colour to an RSOS degree of freedom.

These are given explicitly by $(\alpha \neq \beta)$

$$
\begin{aligned}
&\left(b_{j}^{ \pm}{ }_{j}^{(\alpha, \beta)}\right)_{\boldsymbol{a}, \boldsymbol{b}}=\left(\prod_{k \neq j} \delta_{a_{k}, b_{k}}\right)\left(\prod_{k \neq j, j+1} \delta_{s_{k}, t_{k}}\right) \\
& A_{a_{j-1}, a_{j}}^{(\beta)} A_{b_{j}, b_{j+1}}^{(\beta)} \delta_{a_{j-1}, b_{j}} \delta_{a_{j}, a_{j+1}} \delta_{s_{j+1}, t_{j}}^{(\alpha)}\left(\prod_{k \neq j} \delta_{a_{k}, b_{k}}\right)\left(\prod_{k \neq j, j+1} \delta_{s_{k}, t_{k}}\right) \\
&\left(b_{j}^{ \pm}{ }_{j}^{(\beta, \alpha)}\right)_{\boldsymbol{a}, \boldsymbol{b}}= \delta_{a_{j-1}, a_{j}} \delta_{b_{j}, b_{j+1}} A_{a_{j-1}, b_{j}}^{(\beta)} A_{a_{j}, a_{j+1}}^{(\beta)} \delta_{s_{j}, t_{j+1}}^{(\alpha)} \\
&\left(e_{j}^{(\alpha, \beta)}\right)_{\boldsymbol{a}, \boldsymbol{b}=}\left(\prod_{k \neq j} \delta_{a_{k}, b_{k}}\right)\left(\prod_{k \neq j, j+1} \delta_{s_{k}, t_{k}}\right) \delta_{a_{j-1}, b_{j}, a_{j+1}} \\
&\left(x^{(\alpha)}\right)^{t_{j+1}} \sqrt{\frac{S_{a_{j}}}{S_{a_{j-1}}} A_{a_{j-1}, a_{j}}^{(\beta)} A_{a_{j}, a_{j+1}}^{(\beta)} \delta_{-t_{j}, t_{j+1}}}
\end{aligned}
$$




$$
\begin{array}{r}
\left(e_{j}^{(\beta, \alpha)}\right)_{\boldsymbol{a}, \boldsymbol{b}}=\left(\prod_{k \neq j} \delta_{a_{k}, b_{k}}\right)\left(\prod_{k \neq j, j+1} \delta_{s_{k}, t_{k}}\right) \delta_{a_{j-1}, a_{j}, a_{j+1}} \\
\left(x^{(\alpha)}\right)^{s_{j+1}} \sqrt{\frac{S_{b_{j}}}{S_{a_{j-1}}}} A_{b_{j-1}, b_{j}}^{(\beta)} A_{b_{j}, b_{j+1}}^{(\beta)} \delta_{-s_{j}, s_{j+1}}
\end{array}
$$

where $\alpha$ denotes the colour of the vertex-type and $\beta$ the colour of the RSOS-type degree of freedom. $\boldsymbol{a}$ and $\boldsymbol{b}$ are given by

$$
\begin{aligned}
\boldsymbol{a} & =\left(a_{0}, s_{1}, a_{1}, s_{2}, a_{2}, \ldots, a_{n}, s_{n+1}, a_{n+1}\right) \\
\boldsymbol{b} & =\left(b_{0}, t_{1}, b_{1}, t_{2}, b_{2}, \ldots, b_{n}, t_{n+1}, b_{n+1}\right)
\end{aligned}
$$

and $\delta_{s, t}^{(\alpha)}$ is defined as 3.8

$$
\delta_{s, t}^{(\alpha)}=\left(\prod_{\gamma \neq \alpha} \delta_{s^{(\gamma)}, t^{(\gamma)}, 0}\right) \delta_{s^{(\alpha)}, t^{(\alpha)}} .
$$

Of course, the mixed representations are related to the unrestricted limit of the RSOS representations (for the $m_{V}$ vertex-type colours) in the same way as the vertex representations. Hence, also the characteristic polynomial equations satisfied by the mixed representation are still the same and are therefore given by eq. (3.31).

\section{Two-Colour Representations and Solutions of the Yang-Baxter Equation}

Some of the representations of the two-colour braid-monoid algebra defined in the previous section actually occur in (critical) solvable RSOS models which are related to (coloured) dense or dilute loop models (see [10, 11, 9]). These models gained considerable interest after dilute RSOS models labelled by Dynkin diagrams were found that admit an off-critical extension which breaks the reflection symmetry of the Dynkin diagram [10]. This means that for these models the elliptic nome acts as a magnetic field rather than being a temperaturelike variable as it is in the usual case. As a result, the dilute models related to the IzerginKorepin [32, 33] (or $\mathrm{A}_{2}^{(2)}$ [19]) vertex model include, for instance, a model which belongs to the same universality class as the Ising model in a magnetic field [10]. We are going to have a closer look at RSOS models which are related to $\mathrm{A}_{2}^{(1)}, \mathrm{A}_{3}^{(1)}, \mathrm{A}_{2}^{(2)}$, and $\mathrm{C}_{2}^{(1)}$ vertex models [19, 9].

The representations of interest for our present purpose are representations of the twocolour algebra where either both graphs $\mathcal{G}^{(1)}$ and $\mathcal{G}^{(2)}$ coincide or where one of those (which without loss of generality we assume to be $\mathcal{G}^{(2)}$ ) is just the Dynkin diagram of the Lie algebra $\mathrm{A}_{2}$. As it turns out, the first case corresponds to RSOS models related to dense two-colour loop models [9] whereas the second case corresponds to dilute models [10, 9]. To obtain critical models whose Boltzmann weights are parametrized by trigonometric functions, the 
graphs have to be Dynkin diagrams of "classical" A-D-E type or of their affine counterparts $\mathrm{A}^{(1)}-\mathrm{D}^{(1)}-\mathrm{E}^{(1)}$.

For the case $\mathcal{G}^{(2)}=\mathrm{A}_{2}$, the above representation simplifies considerably. The PerronFrobenius eigenvector is just given by $S_{1}^{(2)}=S_{2}^{(2)}=1$. Furthermore, one has $\Lambda^{(2)}=1$ and hence $x^{(2)}=\exp ( \pm \pi i / 3)$. This yields $i \sqrt{x^{(2)}}=\exp (2 \pi i / 3)\left(\operatorname{resp} . i \sqrt{x^{(2)}}=\exp (\pi i / 3)\right)$ and it follows that

$$
e_{j}^{(2,2)}=\mp b_{j}^{(2,2)}=\mp b_{j}^{-(2,2)}=p_{j}^{(2,2)}
$$

or, in other words, $\sqrt{Q^{(2)}}=1, \omega^{(2)}=\mp 1, f^{(2)}(z)=z \pm 1$, and $g^{(2)}(z)=\mp z$. In order to comply with the usual notation (which is $\lambda^{(\alpha)}=\pi /(L+1)$ for $\mathcal{G}^{(\alpha)}=\mathrm{A}_{L}$ ) we choose the first (upper) sign, i.e. in particular $\omega^{(2)}=-1$. Actually, since the sign of the braids is not fixed by eq. (2.5) this is nothing but a convention.

In addition, we discuss several examples of two-colour vertex models and two-colour mixed vertex-RSOS models. These models are related to the unrestricted SOS models of the RSOS models mentioned above. That means in particular that the algebraic description of the vertex respective vertex-RSOS models is the same as for the corresponding RSOS models, the only difference being that one deals with two different representations of the two-colour braid-monoid algebra.

Our algebraic approach clearly shows that the several models listed above, albeit looking quite different from each other, are in fact closely related and hence provides a uniform characterization of this type of models. On the other hand, we are able to show that the YangBaxter equations for these models follow from the algebraic structure alone (see sec. 4.6). This means that for any representation of the two-colour algebra with the corresponding properties one obtains a solution of the Yang-Baxter equations and hence an exactly solvable model. A more precise formulation as a theorem is presented at the end of this section. Finally, the common algebraic structure strongly suggests a plausible ansatz to find new solvable models. We will come back to this point in our conclusions.

\subsection{Local Face Operator and the Yang-Baxter Equation}

Let us (very briefly) recall the main properties of exactly solvable two-dimensional statistical systems which are defined on a square lattice. For our purpose, it turns out to be convenient to think of the square lattice diagonally as depicted below

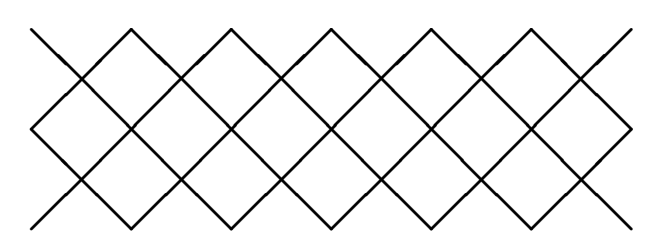

The models are now defined by specifying the degrees of freedom (which usually live on the edges or on the vertices of the lattice) and their interactions.

There are two frequently studied types of models which are the vertex models and the so-called IRF (interaction-round-a-face) models. For vertex models, the degrees of freedom 
("arrows" or "spins") are located on the edges and the interaction takes place at the vertices of the lattice. We denote the Boltzmann weight of a vertex as follows

$$
W\left(\begin{array}{cc|c}
w & v & u \\
s & t & u
\end{array}\right)=\underbrace{w}_{s}
$$

where here and in what follows, the letter $u$ is used for the spectral parameter. On the other hand, IRF models have their degrees of freedom (usually called "heights") situated on the vertices. Here, the interaction takes place between the four corners of an elementary plaquette of the lattice. The Boltzmann weights for such a plaquette have the form

$$
W\left(\begin{array}{c|c}
d & b \\
a & u
\end{array}\right)=d\left\langle\searrow_{a}^{c} b \quad .\right.
$$

Of course, one can also consider face models which are a combination of both. For simplicity, we will not give explicit expression for these models which can be handled in a completely analogous way.

We now introduce face transfer operators (also called Yang-Baxter operators) that act on the configuration along a row of the diagonal lattice. For IRF models, the face transfer operator $X_{j}(u)$ has matrix elements [34, 35]

$$
\left(X_{j}(u)\right)_{\boldsymbol{a}^{\prime}, \boldsymbol{a}}=W\left(a_{j-1} a_{a_{j}}^{\prime} a_{j+1} \mid u\right) \prod_{k \neq j} \delta_{a_{k}, a_{k}^{\prime}}
$$

where

$$
\begin{aligned}
\boldsymbol{a} & =\left(a_{0}, a_{1}, a_{2}, \ldots, a_{n}, a_{n+1}\right) \\
\boldsymbol{a}^{\prime} & =\left(a_{0}^{\prime}, a_{1}^{\prime}, a_{2}^{\prime}, \ldots, a_{n}^{\prime}, a_{n+1}^{\prime}\right)
\end{aligned}
$$

denote allowed height configurations on adjacent rows and $n$ is the size of of the diagonal lattice. Here, "allowed" means that we consider only those configurations which can be extended to a configuration on the whole lattice that has a non-zero Boltzmann weight. For vertex models, the face operators have the following matrix elements 36, 37

$$
\left(X_{j}(u)\right)_{\boldsymbol{s}^{\prime}, \boldsymbol{s}}=W\left(\begin{array}{cc|c}
s_{j} & s_{j}^{\prime} \\
s_{j+1} & s_{j+1}^{\prime}
\end{array} \mid u\right) \prod_{k \neq j, j+1} \delta_{s_{k}, s_{k}^{\prime}} .
$$

Here, $\boldsymbol{s}=\left(s_{1}, s_{2}, \ldots, s_{n}, s_{n+1}\right)$ and $\boldsymbol{s}^{\prime}=\left(s_{1}^{\prime}, s_{2}^{\prime}, \ldots, s_{n}^{\prime}, s_{n+1}^{\prime}\right)$ denote arrow configurations along rows of the diagonal lattice. In fact, the Yang-Baxter operator of a vertex model can be written as a direct product

$$
X_{j}(u)=I \otimes I \otimes \ldots \otimes X(u) \otimes \ldots \otimes I
$$


where $I$ denotes the identity matrix and the local operator $X(u)$ covers the $j$ and $j+1$ slots. In both cases, the Yang-Baxter operators $X_{j}(u)$ are local operators in the sense that, except in the vicinity of site $j$, they act as the identity.

If we assume that the Boltzmann weights (4.3) resp. (4.4) satisfy the Yang-Baxter equations, the corresponding local face operators $X_{j}(u)$ (eq. (4.5) resp. eq. (4.6)) generate the so-called Yang-Baxter algebra [22, 37, 38]

$$
\begin{aligned}
& X_{j}(u) X_{j+1}(u+v) X_{j}(v)=X_{j+1}(v) X_{j}(u+v) X_{j+1}(u) \\
& X_{j}(u) X_{k}(v)=X_{k}(v) X_{j}(u) \quad \text { for }|j-k|>1
\end{aligned}
$$

The first equation essentially is the Yang-Baxter equation, the second follows from the local action of the Yang-Baxter operators. The Yang-Baxter equations guarantee the solvability of the model since it follows that the row transfer matrices of the model form a commuting one-parameter family which yields an infinite number of conserved quantities. Usually, one requires that the Boltzmann weights have additional properties besides fulfilling the YangBaxter equations. Two of them, the standard initial condition (value of Boltzmann weights at $u=0$ ) and the inversion relation (unitarity condition), can be formulated directly in terms of the face transfer operators. They result in the relations

$$
\begin{array}{ll}
X_{j}(0)=I & \text { (Standard Initial Condition) } \\
X_{j}(u) X_{j}(-u)=\varrho(u) \varrho(-u) I & \text { (Inversion Relation) }
\end{array}
$$

where $\varrho(u)$ is a model-dependent function of the spectral parameter. Note that the inversion relation can actually be derived from the Yang-Baxter equation and the standard initial condition.

Another important property of many exactly solvable models is the so-called crossing symmetry. Since it cannot be simply described in terms of the local face operators, we go back to the Boltzmann weights. For IRF models, crossing symmetry means

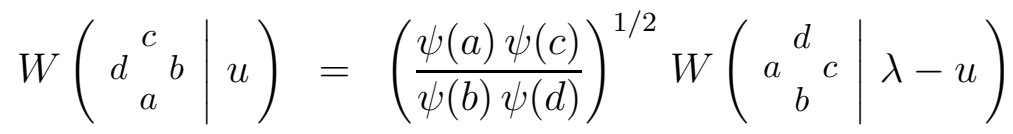

and for vertex models, one has

$$
W\left(\begin{array}{cc|c}
w & v & u \\
s & t & u
\end{array}\right)=\left(\frac{r(s) r(w)}{r(t) r(v)}\right)^{1 / 2} W\left(\begin{array}{cc}
\bar{s} & w \\
t & \bar{v}
\end{array} \mid \lambda-u\right) .
$$

Here, $\lambda$ is the crossing parameter and $\psi(a)$ resp. $r(s)$ are crossing multipliers [34, 37, 38] which are complex numbers and do not depend on the spectral parameter. For the vertex models, $\bar{s}=-s$ means the charge conjugated state of $s$ and the crossing multipliers satisfy $r(\bar{s})=1 / r(s)$. The presence of crossing multipliers means that the Boltzmann weights are not invariant under rotations in general. However, the crossing multipliers of adjacent vertices resp. plaquettes cancel each other and on a periodic lattice (which means periodic in both directions) they do not enter into the partition function at all. This is an example of the more general concept of gauge equivalence. Two models are gauge-equivalent if their 
Boltzmann weights are related by a gauge transformation. In the present context, these are transformations of the Boltzmann weights which do not change the partition function of the model on a lattice which is periodic in both directions. A general local gauge transformation therefore has the form

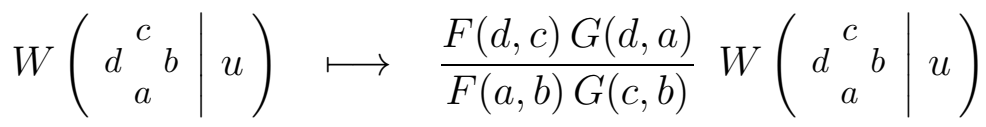

for IRF models and

$$
W\left(\begin{array}{cc|c}
w & v & u \\
s & t & u
\end{array}\right) \frac{F(w, v) G(w, s)}{F(s, t) G(v, t)} W\left(\begin{array}{r}
w^{v} t \\
s
\end{array} \mid u\right)
$$

for vertex models. In both cases, $F$ and $G$ can be arbitrary functions which also are allowed to depend on the spectral parameter $u$. Of course, quantities which are not determined by the partition function on the periodic lattice alone may depend on the particular gauge. For instance, the Yang-Baxter algebra of a solvable model can depend on the gauge. For an example, see the discussion of the $\mathrm{A}_{2}^{(2)}$ vertex model below.

\subsection{RSOS Models labelled by the pair $\left(\mathcal{G}, \mathrm{A}_{2}\right)$}

The two examples of solvable RSOS models we want to consider here are related to the $\mathrm{A}_{2}^{(1)}$ resp. $\mathrm{A}_{2}^{(2)}$ vertex models [19]. As these models are defined in refs. [10] and [9], they are labelled by one simple graph $\mathcal{G}$ (which is of A-D-E type for critical models) and in addition the height variable is allowed to stay at the same value for adjacent sites, i.e. the actual adjacency diagram $\tilde{\mathcal{G}}$ is obtained from $\mathcal{G}$ by affixing a circle at each node of $\mathcal{G}$. For $\mathcal{G}=\mathrm{A}_{L}$ for instance, $\tilde{\mathcal{G}}$ thus looks as follows:

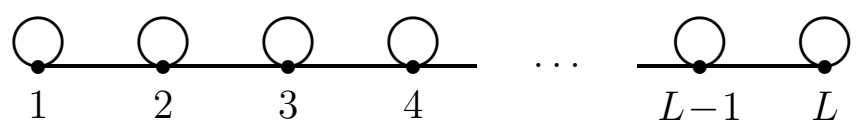

In order to interpret this model as an RSOS model labelled by a pair $\left(\mathcal{G}, \mathrm{A}_{2}\right)$ of graphs, we double the number of heights. That is instead $a \in \mathcal{G}$ we have two heights $(a, b) \in\left(\mathcal{G}, \mathrm{A}_{2}\right)$ with $b \in\{1,2\}$ and hence we have two copies of the graph $\mathcal{G}$. The circles are now replaced by lines connecting the heights $(a, 1)$ and $(a, 2)$ in the two different copies. Thus what we get is the graph $\mathcal{G} \times \mathrm{A}_{2}$ which for our above example $\mathcal{G}=\mathrm{A}_{L}$ looks as follows:

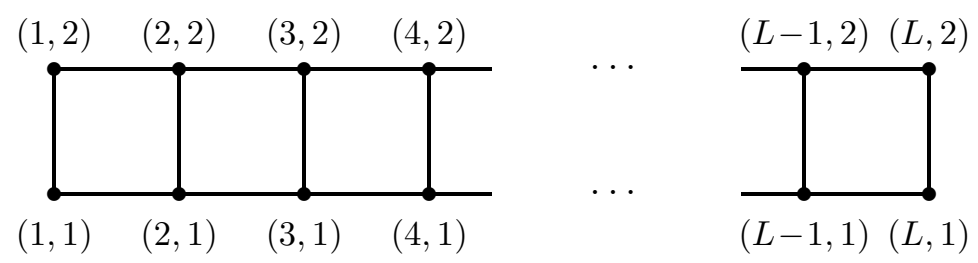


The graph $\mathcal{G} \times \mathrm{A}_{2}$ possesses a natural $\mathbb{Z}_{2}$ symmetry $((a, b) \longmapsto(a, \bar{b}))$ with $\bar{b}=3-b$ and by taking the quotient

$$
\left(\mathcal{G} \times \mathrm{A}_{2}\right) / \mathbb{Z}_{2} \cong \tilde{\mathcal{G}}
$$

one recovers the graph $\tilde{\mathcal{G}}$. This, of course, is a rather trivial example of the orbifold duality of Fendley and Ginsparg [39].

There is an exact one-to-two correspondence between " $\tilde{\mathcal{G}}$-allowed" faces and " $\mathcal{G} \times \mathrm{A}_{2^{-}}$ allowed" faces

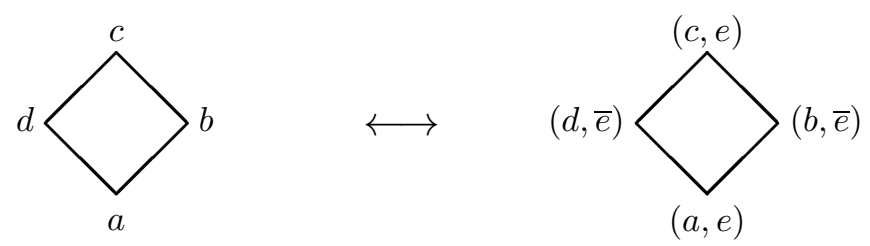

with $e \in\{1,2\}$ and $\bar{e}=3-e$. It is therefore obvious that for any " $\tilde{\mathcal{G}}$-allowed" configuration on a square lattice there are exactly two " $\mathcal{G} \times \mathrm{A}_{2}$ "-allowed configurations. Hence choosing the face weights to be

$$
W\left(\begin{array}{cc}
(d, 2) & (c, 1) \\
(a, 1) & (b, 2)
\end{array} \mid u\right)=W\left(\begin{array}{cc}
(d, 1) & (c, 2) \\
(a, 2) & (b, 1)
\end{array} \mid u\right)=W\left(\begin{array}{cc}
d & c \\
a & b
\end{array} \mid u\right)
$$

one obtains a solvable model with adjacency graph $\mathcal{G} \times \mathrm{A}_{2}$ whose partition function is just twice the partition function of the corresponding dilute model with adjacency graph $\tilde{\mathcal{G}}$.

As our first example, we consider the dilute model which is related to the $\mathrm{A}_{2}^{(1)}$ vertex model [9]. The local face operator (4.5) for this model can be written as follows

$$
\begin{aligned}
X_{j}(u)= & \frac{\sin (\lambda-u)}{\sin (\lambda)}\left(p_{j}^{(1,1)}+p_{j}^{(2,2)}\right)+\left(p_{j}^{(1,2)}+p_{j}^{(2,1)}\right) \\
& +\frac{\sin (u)}{\sin (\lambda)}\left(e_{j}^{(1,1)}+b_{j}^{(1,2)}+b_{j}^{(2,1)}\right)
\end{aligned}
$$

where the braid and monoid operators are understood to be the matrices of the representation labelled by $\left(\mathcal{G}, \mathrm{A}_{2}\right)$ and $\lambda=\lambda^{(1)}$. Obviously, the model is not crossing symmetric since $p_{j}^{(1,2)}$ and $p_{j}^{(2,1)}$ enter in $X_{j}(u)$ with a coefficient one whereas the corresponding "crossed" objects $e_{j}^{(1,2)}$ and $e_{j}^{(2,1)}$ do not show up in the expression for $X_{j}(u)$ at all.

The second example is the dilute model related to the $\mathrm{A}_{2}^{(2)}$ vertex model (Izergin-Korepin model) [10, 9]. Its Yang-Baxter operator (4.5) has the form

$$
\begin{aligned}
X_{j}(u)= & \frac{\sin (2 \bar{\lambda}-u) \sin (3 \bar{\lambda}-u)}{\sin (2 \bar{\lambda}) \sin (3 \bar{\lambda})} p_{j}^{(1,1)}+\left(1+\frac{\sin (u) \sin (3 \bar{\lambda}-u)}{\sin (2 \bar{\lambda}) \sin (3 \bar{\lambda})}\right) p_{j}^{(2,2)} \\
& +\frac{\sin (3 \bar{\lambda}-u)}{\sin (3 \bar{\lambda})}\left(p_{j}^{(1,2)}+p_{j}^{(2,1)}\right) \\
& -\frac{\sin (u) \sin (\bar{\lambda}-u)}{\sin (2 \bar{\lambda}) \sin (3 \bar{\lambda})} e_{j}^{(1,1)}+\frac{\sin (u)}{\sin (3 \bar{\lambda})}\left(e_{j}^{(1,2)}+e_{j}^{(2,1)}\right)
\end{aligned}
$$




$$
+\frac{\sin (u) \sin (3 \bar{\lambda}-u)}{\sin (2 \bar{\lambda}) \sin (3 \bar{\lambda})}\left(b_{j}^{(1,2)}+b_{j}^{(2,1)}\right)
$$

with $\bar{\lambda}=\bar{\lambda}^{(1)}$.

As one recognizes from this expression for the local face operator $X_{j}(u)$, the face weights are manifestly crossing symmetric. The crossing parameter of the model is $3 \bar{\lambda}$.

We choose this model as our main example since it is particularly interesting. Not only does the $\mathcal{G}=\mathrm{A}_{L}$ model with $L$ odd allow an off-critical extension which breaks the reflection symmetry of the Dynkin diagram $\mathrm{A}_{L}$ [10], but the Yang-Baxter algebra (4.8) of the critical model is also non-commuting at one site:

$$
\left[X_{j}(u), X_{j}(v)\right] \neq 0
$$

Of course, this implies that the "full" braid $B_{j}^{ \pm}$obtained from

$$
\begin{aligned}
B_{j}^{ \pm} & =-y^{\mp 2} \lim _{u \rightarrow \mp i \infty} \frac{X_{j}(u)}{\varrho(u)} \\
& =-y^{\mp 2} p_{j}^{(1,1)}-y^{ \pm 2} e_{j}^{(1,1)}+\left(p_{j}^{(2,2)}+b_{j}^{(1,2)}+b_{j}^{(2,1)}\right) \\
& =b_{j}^{ \pm(1,1)}+b_{j}^{ \pm}{ }_{j}^{(1,2)}+b_{j}^{ \pm(2,1)}-b_{j}^{ \pm(2,2)}
\end{aligned}
$$

with $y=\exp (i \bar{\lambda}), \quad b_{j}^{(2,2)}=-p_{j}^{(2,2)}$ (cf. eq. (4.1)) and the "full" monoid $E_{j}$ which is given by

$$
E_{j}=X_{j}(3 \bar{\lambda})=e_{j}^{(1,1)}+e_{j}^{(1,2)}+e_{j}^{(2,1)}+p_{j}^{(2,2)}=e_{j}^{(1,1)}+e_{j}^{(1,2)}+e_{j}^{(2,1)}+e_{j}^{(2,2)}
$$

do not commute. Here,

$$
\varrho(u)=\frac{\sin (2 \bar{\lambda}-u) \sin (3 \bar{\lambda}-u)}{\sin (2 \bar{\lambda}) \sin (3 \bar{\lambda})}
$$

is the function that enters in the inversion relation (4.9). It is therefore obvious that this model cannot be described in terms of the usual (one-colour) braid-monoid algebra. Furthermore, in the general case we were not able to find a gauge transformation (4.12) of the face weights which results in an equivalent model (i.e., a model with the same partition function on a torus) with a commuting algebra at one $\operatorname{site}^{5}$.

It is not possible to express the local face operator $X_{j}(u)$ in terms of the "full" braids and monoids alone. One has to add at least one additional operator, which for instance one can choose to be

$$
F_{j}=p_{j}^{(1,1)}+e_{j}^{(1,1)}+p_{j}^{(2,2)}+e_{j}^{(2,2)}=p_{j}^{(1,1)}+e_{j}^{(1,1)}+2 p_{j}^{(2,2)} .
$$

This yields

$$
X_{j}(u)=\frac{\sin (3 \bar{\lambda}-u)}{\sin (3 \bar{\lambda})} I+\frac{\sin (u)}{\sin (3 \bar{\lambda})} E_{j}
$$

\footnotetext{
${ }^{5}$ We found such a gauge for the model labelled by $\mathcal{G}=\mathrm{A}_{L}^{(1)}$, compare the remarks on the gauge of the $\mathrm{A}_{2}^{(2)}$ vertex model below.
} 


$$
\begin{aligned}
& +\frac{\sin \left(\frac{u}{2}\right) \sin \left(\frac{3 \bar{\lambda}-u}{2}\right)}{\sin (2 \bar{\lambda}) \sin (3 \bar{\lambda})}\left\{\cos \left(\frac{3 \bar{\lambda}}{2}\right)\left(B_{j}+B_{j}^{-1}\right)\right. \\
& \left.+\left(e^{-i\left(\frac{3 \bar{\lambda}}{2}-u\right)} B_{j}+e^{i\left(\frac{3 \bar{\lambda}}{2}-u\right)} B_{j}^{-1}\right)\right\} \\
& +\left(1-\frac{\sin (3 \bar{\lambda}-u)}{\sin (3 \bar{\lambda})}-\frac{\sin (u)}{\sin (3 \bar{\lambda})}\right) F_{j} .
\end{aligned}
$$

The relations for the "full" braids and monoids and the additional operator $F_{j}$ can be computed from the relations for the coloured operators. As it turns out, the "full" braid $B_{j}$ (4.22) satisfies a quartic equation

$$
\left(B_{j}-I\right)\left(B_{j}+I\right)\left(B_{j}+y^{-2} I\right)\left(B_{j}-y^{6} I\right)=0
$$

whereas the "full" monoid $E_{j}(4.23)$ fulfills the quadratic

$$
\left(E_{j}\right)^{2}=\left(1-y^{4}-y^{-4}\right) E_{j}=(1-2 \cos (4 \bar{\lambda})) E_{j}
$$

and the additional operator $F_{j}(4.25)$ satisfies another quartic

$$
F_{j}\left(F_{j}-I\right)\left(F_{j}-2 I\right)\left(F_{j}-\left(1-y^{4}-y^{-4}\right) I\right)=0 .
$$

The commutator of the braid and the monoid,

$$
C_{j}=\frac{1}{y^{ \pm 6}-1}\left[B_{j}^{ \pm}, E_{j}\right]
$$

fulfills a simple cubic relation

$$
C_{j}\left(C_{j}-I\right)\left(C_{j}+I\right)=0 .
$$

Furthermore, one finds the following commutation relations

$$
\left[F_{j}, B_{j}^{ \pm}\right]=0,\left[F_{j}, E_{j}\right]=-\left(1+y^{4}+y^{-4}\right) C_{j},
$$

to present just a few of the many relations that one can derive from the two-colour algebra.

So far, we only considered some relations of operators at the same site. It turns out that the "full" braids and monoids in fact fulfill all defining relations of the one-colour braid-monoid algebra except the twist relations. In particular, the one-colour braid-monoid relations (2.5) are satisfied. This actually follows from the third remark we made in the beginning of this section (see eqs. (3.9) and (3.10). However, we want to emphasize again that neither $E_{j}$ nor $F_{j}$ is a polynomial in the braid $B_{j}$. To describe the algebra completely in terms of $B_{j}, E_{j}$, and $F_{j}$, one needs of course additional relations with operators acting on tho neighbouring sites which involve the operator $F_{j}$ and which can be derived from the corresponding relations of the two-colour algebra.

As we demonstrated, one can alternatively describe the local Yang-Baxter algebra of the dilute model in terms of the (spectral-parameter independent) operators $B_{j}, E_{j}$, and $F_{j}$. 
However, it should be clear from this exercise that the formulation using the two-colour braid-monoid algebra has several advantages. It is simpler and certainly more natural in the sense that the two-colour algebra is a straight-forward generalization of the Temperley-Lieb algebra and that it still allows a graphical interpretation and therefore is related to properties of coloured knots and links.

\subsection{RSOS Models labelled by the pair $(\mathcal{G}, \mathcal{G})$}

Again, we present two examples one of which consists of a series of crossing symmetric models. The models considered here are the dense two-colour loop models of ref. [9]. We are going to have a closer look at the RSOS models related to $\mathrm{C}_{2}^{(1)}$ since these models are crossing symmetric.

First, let us have a short glance at an IRF model related to the $\mathrm{A}_{3}^{(1)}$ vertex model [9]. The Yang-Baxter operator (4.5) for the model reads as follows

$$
\begin{aligned}
X_{j}(u)= & \frac{\sin (\lambda-u)}{\sin (\lambda)}\left(p_{j}^{(1,1)}+p_{j}^{(2,2)}\right)+\left(p_{j}^{(1,2)}+p_{j}^{(2,1)}\right) \\
& +\frac{\sin (u)}{\sin (\lambda)}\left(e_{j}^{(1,1)}+e_{j}^{(2,2)}+b_{j}^{(1,2)}+b_{j}^{(2,1)}\right)
\end{aligned}
$$

where $\lambda=\lambda^{(1)}=\lambda^{(2)}$ is determined by eq. (3.20). As the models defined by eq. (4.19) above, this model does not possess a crossing symmetry. Again, the two-colour monoids $e_{j}^{(\alpha, \beta)}(\alpha \neq \beta)$ do not enter in the expression for the local face operator at all.

We are more interested in our second example which is the above mentioned IRF model related to the $\mathrm{C}_{2}^{(1)}$ vertex model [9]. The local face operator (4.5) of this model has the form

$$
\begin{aligned}
X_{j}(u)= & \frac{\sin (2 \bar{\lambda}-u) \sin (6 \bar{\lambda}-u)}{\sin (2 \bar{\lambda}) \sin (6 \bar{\lambda})}\left(p_{j}^{(1,1)}+p_{j}^{(2,2)}\right)+\frac{\sin (6 \bar{\lambda}-u)}{\sin (6 \bar{\lambda})}\left(p_{j}^{(1,2)}+p_{j}^{(2,1)}\right) \\
& -\frac{\sin (u) \sin (4 \bar{\lambda}-u)}{\sin (2 \bar{\lambda}) \sin (6 \bar{\lambda})}\left(e_{j}^{(1,1)}+e_{j}^{(2,2)}\right)+\frac{\sin (u)}{\sin (6 \bar{\lambda})}\left(e_{j}^{(1,2)}+e_{j}^{(2,1)}\right) \\
& +\frac{\sin (u) \sin (6 \bar{\lambda}-u)}{\sin (2 \bar{\lambda}) \sin (6 \bar{\lambda})}\left(b_{j}^{(1,2)}+b_{j}^{(2,1)}\right)
\end{aligned}
$$

with $\bar{\lambda}=\bar{\lambda}^{(1)}=\bar{\lambda}^{(2)}$ (cf. eq. (3.21)). Here, the crossing symmetry is manifestly built-in again, with crossing parameter $6 \bar{\lambda}$.

In contrast to the previous crossing-symmetric example (4.20), the local face operator is still commuting at one site, i.e.,

$$
\left[X_{j}(u), X_{j}(v)\right]=0
$$

This stems from the fact that the model is labelled by two copies of the same graph and hence the twists $\omega^{(1)}$ and $\omega^{(2)}$ coincide which guarantees the commutativity of the "full" braid and monoid. Still, one cannot express the local face operator $X_{j}(u)$ entirely in terms of the "full" 
braids and monoids. One again has to introduce (at least) one additional operator, which in the present case can be chosen as

$$
F_{j}=b_{j}^{(1,2)}+b_{j}^{(2,1)}
$$

for instance. For the local face operator, one obtains

$$
\begin{aligned}
& X_{j}(u)= \frac{\sin (6 \bar{\lambda}-u)}{\sin (6 \bar{\lambda})} I+\frac{\sin (u)}{\sin (6 \bar{\lambda})} E_{j} \\
&+\frac{\sin \left(\frac{u}{2}\right) \sin \left(\frac{6 \bar{\lambda}-u}{2}\right)}{\sin (2 \bar{\lambda}) \sin (6 \bar{\lambda})}\left\{\frac{\cos (\bar{\lambda})}{\cos (2 \bar{\lambda})}\left(B_{j}+B_{j}^{-1}\right)\right. \\
&\left.+\left(e^{-i\left(\frac{6 \bar{\lambda}}{2}-u\right)} B_{j}+e^{i\left(\frac{6 \bar{\lambda}}{2}-u\right)} B_{j}^{-1}\right)\right\} \\
&+\frac{1}{2 \cos (2 \bar{\lambda})}\left(1-\frac{\sin (6 \bar{\lambda}-u)}{\sin (6 \bar{\lambda})}-\frac{\sin (u)}{\sin (6 \bar{\lambda})}\right) F_{j}
\end{aligned}
$$

which shows striking similarity to eq. (4.26). Here, the "full" braids and monoids are given by

$$
\begin{aligned}
& B_{j}^{ \pm}=-y^{\mp 2} \lim _{u \rightarrow \mp i \infty} \frac{X_{j}(u)}{\varrho(u)}=\sum_{\alpha, \beta} b_{j}^{ \pm(\alpha, \beta)} \\
& E_{j}=X_{j}(6 \bar{\lambda})=\sum_{\alpha, \beta} e_{j}^{(\alpha, \beta)}
\end{aligned}
$$

where

$$
\varrho(u)=\frac{\sin (2 \bar{\lambda}-u) \sin (6 \bar{\lambda}-u)}{\sin (2 \bar{\lambda}) \sin (6 \bar{\lambda})}
$$

is the function that enters in the inversion relation (4.9). The following relations hold

$$
\begin{aligned}
& \left(B_{j}-I\right)\left(B_{j}+I\right)\left(B_{j}+y^{-2} I\right)\left(B_{j}-y^{6} I\right)=0 \\
& \left(E_{j}\right)^{2}=-2\left(y^{4}+y^{-4}\right) E_{j}=-4 \cos (4 \bar{\lambda}) E_{j} \\
& B_{j} E_{j}=E_{j} B_{j}=y^{6} E_{j} \\
& F_{j}\left(F_{j}+I\right)\left(F_{j}-I\right)=0 \\
& {\left[B_{j}^{ \pm}, F_{j}\right]=\left[E_{j}, F_{j}\right]=0 .}
\end{aligned}
$$

Obviously, this very much resembles a representation of the one-colour braid-monoid algebra where the braid satisfies a fourth order polynomial equation. However, the monoid $E_{j}$ as well as the additional operator $F_{j}$ cannot be written as a polynomial in the braid $B_{j}$. 


\subsection{Vertex Models of type $(6-\mathrm{V}, 6-\mathrm{V})$}

We are going to mention two models which fall into this category: the $\mathrm{A}_{3}^{(1)}$ and $\mathrm{C}_{2}^{(1)}$ vertex models 19. These are of course equivalent to the unrestricted case of the two series of RSOS models defined above.

This implies that the Yang-Baxter operators of the $\mathrm{A}_{3}^{(1)}$ and $\mathrm{C}_{2}^{(1)}$ vertex models are given by the very same expressions as those of the related face models, i.e. by eq. (4.33) for the $A_{3}^{(1)}$ vertex model and by eq. (4.34) for the $\mathrm{C}_{2}^{(1)}$ case. The only difference is that the representation of the two-colour braid-monoid algebra is now of vertex-type $(6 \mathrm{~V}, 6 \mathrm{~V})$ with $\lambda^{(1)}=\lambda^{(2)}=\lambda$ and $\bar{\lambda}=(\lambda \pm \pi) / 4$ or $\bar{\lambda}=(\lambda \pm 3 \pi) / 4$. It is given explicitly by eqs. (3.11) $-(3.17)$ above.

Of course, the results on the "full" braids and monoids for the RSOS models related to $\mathrm{C}_{2}^{(1)}$ (see eqs. (4.34)-(4.39)) also carry over to the vertex model. This may surprise many readers since the Yang-Baxter algebra of the $\mathrm{C}_{2}^{(1)}$ vertex model usually is known to be a Birman-Wenzl-Murakami (BWM) algebra [6, 7], hence a one-colour braid-monoid algebra where the braids satisfy a third order polynomial equation. However, the vertex model we consider here differs from the $\mathrm{C}_{2}^{(1)}$ vertex model of Jimbo 19 by a spectral-parameter dependent gauge transformation (4.13) which affects the braid limit and therefore influences the algebraic relations. This is well-known, see e.g. eq. (6.12) of ref. [8] where such a gauge transformation is used in order to obtain an "interesting" braid group representation from the six-vertex model. The same happens in the case of the $\mathrm{A}_{2}^{(2)}$ vertex model which is going to be discussed subsequently.

\subsection{Mixed Vertex-RSOS Models}

Here, we again have two examples which are linked to the RSOS models considered above, namely the $\mathrm{A}_{2}^{(1)}$ and $\mathrm{A}_{2}^{(2)}$ vertex models 19 which are related to representations of type $\left(6-\mathrm{V}, \mathrm{A}_{2}\right)$. As above, these models are equivalent to the unrestricted case of the two corresponding series of RSOS models defined in eqs. (4.19) and (4.20). Hence the expressions for the Yang-Baxter operator of the $\mathrm{A}_{2}^{(1)}$ and $\mathrm{A}_{2}^{(2)}$ vertex models again coincide with those of the related face models which are given in eq. (4.19) for the $\mathrm{A}_{2}^{(1)}$ models and in eq. (4.20) for the $\mathrm{A}_{2}^{(2)}$ models, respectively. The corresponding representation of the two-colour braid-monoid algebra is now the mixed representation which we called $\left(6-\mathrm{V}, \mathrm{A}_{2}\right)$ (see eqs. (3.34)-(3.37) above).

For the $\mathrm{A}_{2}^{(2)}$ vertex model, also known under the name Izergin-Korepin model [32, 33], the same comment applies as for the $\mathrm{C}_{2}^{(1)}$ vertex model before. Again, the vertex model obtained as the unrestricted case of the dilute $\mathrm{A}-\mathrm{D}-\mathrm{E}$ models differs from the $\mathrm{A}_{2}^{(2)}$ vertex model of refs. [32, 19, 33] by a spectral-parameter dependent gauge (4.13). Of course, we posed ourselves the question if one can get a one-site commuting Yang-Baxter algebra for the dilute A-D-E models by an appropriate spectral-parameter dependent gauge transformation (4.12). It turns out that this is at least possible for the dilute $A_{L}^{(1)}$ models where the gauge transformation of the vertex model carries over directly. For the other cases, notably for the most interesting case of the dilute $\mathrm{A}_{L}$ models, we did not succeed to find such a transformation (although we also tried to use the additional gauge freedom that one gains by the doubling of the states we did in order to interpret the dilute models as two-colour 
models) and it is our opinion that at least a local gauge of the form (4.12) does not exist.

Recent results of S. O. Warnaar and B. Nienhuis [9] suggest that there are in fact several infinite series of mixed vertex-RSOS models. These include models with an arbitrary number of colours, but all of them (except one or two) occur in the vertex-type representation. As an explicit example, they consider the $\mathrm{A}_{3}^{(2)}$ loop-vertex model which in our language would correspond to a mixed vertex-RSOS model of type $(6-\mathrm{V}, \mathcal{G})$ which however is not crossing symmetric.

In [9], these models are obtained by a partial mapping of well-known vertex models 19 to loop models. The loop degrees of freedom in turn can be rewritten as an RSOS model (in the sense that their partition functions on an infinite lattice coincide, for more details see [9]). The vertex models investigated in [9] in this context are the $\mathrm{A}_{n}^{(1)}, \mathrm{A}_{n}^{(2)}$, and $\mathrm{C}_{n}^{(1)}$ vertex models [19].

\subsection{Yang-Baxterization}

So far, we presented several examples of critical exactly solvable models whose local YangBaxter operators can be written in terms of two-colour braid-monoid operators in certain matrix representations. Now, we address the question to which extent the algebraic structure is responsible for the solvability of the model, i.e. in particular for the inversion relation and the Yang-Baxter equation. Let us formulate the result as a theorem:

Theorem: Let $P_{j}^{(\alpha)}, b_{j}^{ \pm}{ }_{j}^{(\alpha, \beta)}$, and $e_{j}^{(\alpha, \beta)}(\alpha=1,2)$ denote a representation of the twocolour braid-monoid algebra and $p_{j}^{(\alpha, \beta)}=P_{j}^{(\alpha)} P_{j}^{(\beta)}$. Then the following statements hold:

(i) If $\sqrt{Q^{(1)}}=2 \cos (\lambda), f^{(1,2)}(z)=f^{(2,1)}(z)=z-1$, and if $X_{j}(u)$ is given by eq. (4.19), then $X_{j}(u)$ generates a Yang-Baxter algebra (4.8) and the inversion relation (4.9) holds with $\varrho(u)=\frac{\sin (\lambda-u)}{\sin (\lambda)}$.

(ii) If $\sqrt{Q^{(1)}}=\sqrt{Q^{(2)}}=2 \cos (\lambda), f^{(1,2)}(z)=f^{(2,1)}(z)=z-1$, and if $X_{j}(u)$ is given by eq. (4.33), then $X_{j}(u)$ generates a Yang-Baxter algebra (4.8) and the inversion relation (4.9) holds with $\varrho(u)=\frac{\sin (\lambda-u)}{\sin (\lambda)}$.

(iii) If $\sqrt{Q^{(1)}}=-2 \cos (4 \bar{\lambda}), \sqrt{Q^{(2)}}=1, f^{(1,2)}(z)=f^{(2,1)}(z)=z-1, f^{(2)}(z)=z+1$, $g^{(2,2)}(z)=-z$, and if $X_{j}(u)$ is given by eq. (4.20), then $X_{j}(u)$ generates a Yang-Baxter algebra (4.8) and the inversion relation (4.9) holds with $\varrho(u)=\frac{\sin (2 \bar{\lambda}-u) \sin (3 \bar{\lambda}-u)}{\sin (2 \bar{\lambda}) \sin (3 \bar{\lambda})}$.

(iv) If $\sqrt{Q^{(1)}}=\sqrt{Q^{(2)}}=-2 \cos (4 \bar{\lambda}), f^{(1,2)}(z)=f^{(2,1)}(z)=z-1$, and if $X_{j}(u)$ is given by eq. (4.34), then $X_{j}(u)$ generates a Yang-Baxter algebra (4.8) and the inversion relation (4.9) holds with $\varrho(u)=\frac{\sin (2 \bar{\lambda}-u) \sin (6 \bar{\lambda}-u)}{\sin (2 \bar{\lambda}) \sin (6 \bar{\lambda})}$.

The proofs consist of lengthy, but straight-forward direct calculations. Note that the values of $\omega^{(\alpha)}$ and the functions $f^{(\alpha)}(z)$ do not always enter since we eliminated all braids $b_{j}^{ \pm}{ }_{j}^{(\alpha, \alpha)}$ in the expressions for the Yang-Baxter operator $X_{j}(u)$. Nevertheless, they are fixed indirectly, since performing a braid limit on these expressions determines the braids in terms of the other operators (up to normalization). 


\section{Conclusions}

We defined a multi-colour braid-monoid algebra as a straight-forward generalization of the one-colour case. The diagrammatic interpretation also generalizes from the one-colour case. This means that there is a direct relation to the theory of coloured knots and links. The notion of crossing symmetry was discussed using the pictorial representation of the algebra. Different general classes of representations which are connected to solvable lattice models were given explicitly. The two-colour algebra turned out to describe the Yang-Baxter algebra of recently constructed solvable models which are related to dilute one-colour loop models [10, 11, 9] and to dense two-colour loop models [9]. For several examples (which were taken from ref. [9]), we expressed the Yang-Baxter operator of the models in terms of generators of the two-colour braid-monoid algebra and our main result is that we could actually prove that the solvability of the model follows from the algebraic structure alone. These include the so-called dilute A-D-E models [10, 11] which are of particular interest since the dilute $\mathrm{A}_{L}$ models with $L$ odd allow an integrable off-critical extension that breaks the symmetry of the Dynkin diagram $A_{L}$. Hence, the physical meaning of the elliptic nome is that of a magnetic field. This has for instance the consequence that, although the two-dimensional Ising model in a magnetic field has not been solved, the dilute $\mathrm{A}_{3}$ model provides us with an exactly solvable model which belongs to the same universality class.

The dilute A-D-E models possess another interesting property. Their Yang-Baxter algebra, which is the algebra generated by the local face operators, is non-commuting at one site. For these models, a description using only "full" braids and monoids (which are obtained essentially by summing up the coloured objects) turned out to be complicated. It involved at least one new object and we doubt that there is a nice diagrammatic interpretation for the relations satisfied by these operators. Our description of the dilute models as two-colour models is certainly more natural. For the rest of our examples, similar observations apply.

As one notices, there is an apparent similarity in the expressions for the Yang-Baxter operators of the RSOS models labelled by $\left(\mathcal{G}, \mathrm{A}_{2}\right)$ (eqs. (4.19)-(4.20)) and of those labelled by $(\mathcal{G}, \mathcal{G})$ (eqs. (4.33)-(4.34)). This suggests that it might be possible to Yang-Baxterize 12 any representation of the two-colour braid-monoid algebra with the same properties as the representation labelled by any pair $\left(\mathcal{G}^{(1)}, \mathcal{G}^{(2)}\right)$ of graphs.

Conjecture: Let $p_{j}^{(\alpha, \beta)}, b_{j}^{ \pm}{ }_{j}^{(\alpha, \beta)}$, and $e_{j}^{(\alpha, \beta)}(\alpha, \beta=1,2)$ be a representation of the twocolour braid monoid algebra with

$$
\begin{aligned}
\sqrt{Q^{(\alpha)}} & =-\left(k^{(\alpha)}\right)^{2}-\left(k^{(\alpha)}\right)^{-2} \\
\omega^{(\alpha)} & =-\left(k^{(\alpha)}\right)^{-3} \\
f^{(\alpha)}(z) & =\left(z-\omega^{(\alpha)}\right)\left(z-k^{(\alpha)}\right) \\
f^{(\alpha, \beta)}(z) & =z-1 \quad(\alpha \neq \beta) \\
g^{(\alpha)}(z) & =k^{(\alpha)}\left(z-k^{(\alpha)}\right),
\end{aligned}
$$


where $k^{(1)}$ and $k^{(2)}$ are arbitrary complex numbers (of modulus one). Then this representation can be Yang-Baxterized to a (critical) solvable model whose Yang-Baxter operator is of the form

$$
\begin{aligned}
X_{j}(u)= & f_{1}(u) p_{j}^{(1,1)}+f_{2}(u) p_{j}^{(2,2)}+f_{3}(u) p_{j}^{(1,2)}+f_{4}(u) p_{j}^{(2,1)} \\
+ & f_{5}(u) e_{j}^{(1,1)}+f_{6}(u) e_{j}^{(2,2)}+f_{7}(u) e_{j}^{(1,2)}+f_{8}(u) e_{j}^{(2,1)} \\
+ & f_{9}(u) b_{j}^{(1,1)}+f_{10}(u) b_{j}^{(2,2)}+f_{11}(u) b_{j}^{(1,2)}+f_{12}(u) b_{j}^{(2,1)}
\end{aligned}
$$

where the coefficient functions $f_{i}(u)$ are products of trigonometric functions of the spectral parameter $u$ involving (in general) both $\lambda^{(1)}$ and $\lambda^{(2)}$.

Even more, one might conjecture that this extends to the $m$-colour case although we are not aware of any known restricted model which is related to the $m$-colour algebra with $m>2$. Work to check these conjectures is currently in progress.

In case these conjectures turn out to be correct, this would provide us with a method to obtain new solvable critical models. In some sense this procedure resembles the fusion (both have a diagrammatic interpretation acting on composite strings, cf. e.g. ref. [8) of solvable models but, as the above examples show clearly, it in fact leads to totally different models.

Furthermore, all examples we presented in this paper in fact correspond to two-colour generalizations of the Temperley-Lieb algebra only. In particular, this means that the coloured braids fulfill quadratic equations. One would certainly expect that one can find representations of the multi-colour algebra and solvable models related to them which for instance generalize the BWM algebra (where the braids fulfill a cubic equation). Hence our results open up a variety of interesting directions for further investigations.

The authors gratefully acknowlegde financial support from the Deutsche Forschungsgemeinschaft (UG) and from the Australian Research Council. We would like to thank B. Nienhuis, K. A. Seaton and S. O. Warnaar for helpful comments and for sending us their results prior to publication. One of us (PAP) would like to thank B. Nienhuis and FOM for hospitality and support during his visit to the University of Amsterdam.

\section{References}

[1] M. Jimbo, Yang-Baxter Equation in Integrable Systems, World Scientific, Singapore, 1989

[2] C. N. Yang and M. L. Ge, Braid Group, Knot Theory and Statistical Mechanics, World Scientific, Singapore, 1989

[3] H. N. V. Temperley and E. H. Lieb, Proc. Roy. Soc. (London) A322 (1971) 251

[4] P. P. Martin, Potts Models and Related Problems in Statistical Mechanics, World Scientific, Singapore, 1991

[5] L. Kauffman, Topology 26 (1987) 395

[6] J. Birman and H. Wenzl, preprint (1987); Trans. Am. Math. Soc. 313 (1989) 249 
[7] J. Murakami, Osaka J. Math. 24 (1987) 745

[8] M. Wadati, T. Deguchi and Y. Akutsu, Phys. Rep. 180 (1989) 247

[9] S. O. Warnaar and B. Nienhuis, Solvable lattice models labelled by Dynkin diagrams, preprint

[10] S. O. Warnaar, B. Nienhuis and K. A. Seaton, Phys. Rev. Lett. 69 (1992) 710

[11] $\mathrm{Ph}$. Roche, On the construction of integrable dilute $A-D-E$ models, preprint

[12] V. F. R. Jones, Int. J. Mod. Phys. B4 (1990) 701

[13] Y. Akutsu and T. Deguchi, Phys. Rev. Lett. 67 (1991) 777

[14] T. Deguchi and Y. Akutsu, J. Phys. Soc. Japan 60 (1991) 2559

[15] T. Deguchi, J. Phys. Soc. Japan 60 (1991) 3978

[16] T. Deguchi and Y. Akutsu, J. Phys. Soc. Japan 60 (1991) 4051

[17] Y. Akutsu, T. Deguchi and T. Wadati, J. Knot Theory and Ram. 1 (1992) 161

[18] Y. Cheng, M.-L. Ge, G. C. Liu and K. Xue, J. Knot Theory and Ram. 1 (1992) 31

[19] M. Jimbo, Commun. Math. Phys. 102 (1986) 537

[20] A. L. Owczarek and R. J. Baxter, J. Stat. Phys. 49 (1987) 1093

[21] P. A. Pearce, Int. J. Mod. Phys. B4 (1990) 715

[22] R. J. Baxter, Exactly Solved Models in Statistical Mechanics, Academic Press, London, 1982

[23] V. Pasquier, Nucl. Phys. B285 (1987) 162

[24] V. Pasquier, J. Phys. A20 (1987) 5707

[25] V. Pasquier, J. Phys. A20 (1987) L1229

[26] G. E. Andrews, R. J. Baxter and P. J. Forrester, J. Stat. Phys. 35 (1984) 193

[27] P. A. Pearce and K. A. Seaton, Phys. Rev. Lett. 60 (1988) 1347

[28] P. A. Pearce and K. A. Seaton, Ann. Phys. (N.Y.) 193 (1989) 326

[29] A. Kuniba and T. Yajima, J. Stat. Phys. 52 (1988) 829

[30] F. Y. Wu, Phys. Rev. B4 (1971) 2312

[31] L. P. Kadanoff and J. Wegner, Phys. Rev. B4 (1971) 3983

[32] A. G. Izergin and V. E. Korepin, Commun. Math. Phys. 79 (1981) 303

[33] V. I. Vichirko and N. Yu. Reshetikhin, Teor. i Mat. Fiz. 56 (1983) 260

[34] Y. Akutsu, T. Deguchi and M. Wadati, J. Phys. Soc. Japan 57 (1988) 1173 
[35] T. Deguchi, M. Wadati and Y. Akutsu, J. Phys. Soc. Japan 57 (1988) 2921

[36] Y. Akutsu and M. Wadati, J. Phys. Soc. Japan 56 (1987) 839

[37] Y. Akutsu and M. Wadati, J. Phys. Soc. Japan 56 (1987) 3039

[38] T. Deguchi, M. Wadati and Y. Akutsu, J. Phys. Soc. Japan 57 (1988) 1905

[39] P. Fendley and P. Ginsparg, Nucl. Phys. B324 (1989) 549 